\title{
Magnetized initial conditions for CMB anisotropies
}

\author{
Massimo Giovannini \\ Department of Physics, Theory Division, CERN, 1211 Geneva 23, Switzerland
}

(Received 24 September 2004; published 3 December 2004)

\begin{abstract}
This paper introduces a systematic treatment of the linear theory of scalar gravitational perturbations in the presence of a fully inhomogeneous magnetic field. The analysis is conducted both in the synchronous and in the conformally Newtonian gauges. The cosmological plasma is assumed to be composed of cold dark matter, baryons, photons, neutrinos. The problem of superhorizon initial conditions for the fluid variables of the various species and for the coupled system of BoltzmannEinstein equations is discussed in the presence of an inhomogeneous magnetic field. The tight-coupling approximation for the Boltzmann hierarchy is extended to the case where gravitating magnetic fields are included.
\end{abstract}

DOI: $10.1103 /$ PhysRevD.70.123507

PACS numbers: $98.80 . \mathrm{Cq}, 95.85 . \mathrm{Sz}, 98.70 . \mathrm{Vc}$

\section{INTRODUCTION}

The impact of magnetic fields on the anisotropies of the Cosmic Microwave Background (CMB) has been a subject of active investigation since the early attempts of Zeldovich [1,2], where it was proposed that all the anisotropy in the CMB could be generated by a magnetic field. Following the development of inflationary cosmology it is now clear that the explanation of the large-scale temperature anisotropies should be attributed to some adiabatic (or quasiadiabatic) mode, which was present outside the horizon prior to the decoupling of radiation from matter.

The theory of scalar gravitational fluctuations is a well developed subject and it is an essential tool setting the initial conditions for the evolution of CMB anisotropies. When initial conditions are set, usually deep within the radiation era (but after neutrino decoupling taking place around $1 \mathrm{MeV}$ ), the dominant components of the plasma are photons and (effectively massless) neutrinos. The subdominant component of the plasma is formed by baryons, electrons, cold dark matter (CDM) particles. CDM particles are only coupled to gravitational interactions and behave like a perfect relativistic fluid. Also the photons, leptons and baryons, being tightly coupled through Thompson scattering, behave like perfect relativistic fluids. On the contrary, neutrinos are essentially collisionless and, therefore, do not really behave like a perfect fluid. Because of this physical difference, neutrinos should be described through the appropriate Boltzmann hierarchy of their phase-space distribution. If the dark energy is parametrized in terms of a cosmological term, the gravitational fluctuations of this sector do not affect the problem of the initial conditions.

If primordial fluctuations of the geometry are present outside the horizon before matter-radiation equality, they imprint also fluctuations in the density contrasts and peculiar velocities of the different species. The quantitative evolution of the various plasma quantities is determined by the fluctuations of the geometry by solving the coupled system of Einstein and fluid equations in the radiation-dominated epoch when the relevant modes are still outside the horizon. Following the classification scheme pioneered by Bardeen [3], the initial conditions may be classified into adiabatic or isocurvature modes. In the adiabatic case the total fluctuations in the entropy density of the CDM-photon-lepton-baryon fluid vanish at large distance scales which is compatible with a constant mode of curvature fluctuations outside the horizon. In the case of isocurvature modes, in the usual terminology, the fluctuations in the entropy density do not vanish; according to the linear analysis of the Einstein-fluid system, this is compatible, in some cases, with nonconstant modes of the curvature fluctuations. In spite of this simplified classification, the situation may be more complicated since different isocurvature modes may be allowed for the different species present in the plasma. Moreover, it is also quite plausible to have a situation where the initial conditions are, predominantly, of adiabatic nature, but with a subdominant isocurvature component.

Although an excellent approximation early on, the tight-coupling assumption breaks down at later times, when photons and baryons decouple. In spite of this caveat, a systematic use of the tight-coupling expansion allows important analytical estimates of the produced CMB anisotropies [4].

In the theory of the CMB anisotropies, the problem of initial conditions is often presented in two complementary descriptions, namely, the conformally Newtonian and the synchronous gauges. Both gauges are quite useful for different reasons (see also [5]). The conformally Newtonian gauge (often dubbed longitudinal) is effective in the discussion of the evolution of the fluctuations while they are still outside the horizon. However, in such a gauge, the discussion of the initial conditions for the CMB anisotropies may be rather complicated. For instance, there are physical isocurvature modes that are singular in the longitudinal gauge but not in the synchro- 
nous gauge. Furthermore, various codes needed for the numerical calculations of the CMB spectra are formulated within the synchronous coordinate system. There have been, in the past, controversies concerning the use of the synchronous gauge [6]. The main caveat was that gauge modes may appear in the synchronous gauge. These gauge modes, however, can be precisely disentangled from the physical ones [6], so that a pragmatic approach envisaged some time ago [5] has been to discuss the problem of initial conditions in both gauges in parallel.

Suppose now that inhomogeneous magnetic fields are present. In this case two major effects can be envisaged:

(i) inhomogeneous magnetic fields can gravitate, i.e., they can affect the perturbed Einstein equations, thus becoming sources of the fluctuations of the geometry;

(ii) inhomogeneous magnetic fields can impart different velocity gradients to the baryon-photon-lepton fluid, but not to the neutrinos and to the CDM components.

Deep within the radiation epoch, large-scale magnetic fields can be treated, in first approximation, as interacting with a single globally neutral fluid. In fact the two electrically charged species present in the problem are the electrons and the baryons which are in thermal equilibrium at a common temperature. The typical length scales of interest for the present discussion are the ones much larger than the typical magnetic diffusivity scale (set by the finite value of the conductivity) and also much larger than the Silk damping scale (set by the finite value of the thermal diffusivity scale). Furthermore, for this range of scales we are not interested in the propagation of high frequency electromagnetic waves whose specific analysis would clearly require a two-fluid plasma treatment. The one-fluid plasma description is often dubbed as magnetohydrodynamics (MHD). In MHD, the current density, the magnetic field and the electric field are all solenoidal and the Lorentz force affecting the baryon peculiar velocity modifies the standard tight-coupling approximation.

By looking at the evolution of the brightness of the temperature fluctuations it is rather clear that, to zeroth order in the tight-coupling expansion, the monopole and dipole equations are modified. It is known [4] that to zeroth order in the tight-coupling expansion the CMB is not linearly polarized since, to this order, photons and baryons are so strongly coupled that the photon distribution is isotropic in the baryon rest frame. The photon distribution being isotropic, Thompson scattering does not polarize the CMB. To first order in the tight-coupling expansion the polarization of the CMB is proportional to the quadrupole of the photon distribution. The quadrupole in the temperature fluctuations is, in turn, produced by the free streaming of the dipole between collisions. If a Lorentz force term is present, the evolution of the monopole acquires a further source term and, as a con- sequence, all the higher orders in the tight-coupling expansion are modified.

Various analyses of the possible impact of magnetic fields on standard CMB physics ${ }^{1}$ have been proposed up to now (see, for instance, [7] for a short review on this specific subject). In Ref. [8] the analysis of scalar fluctuations of the geometry is made under the assumption that the Lorentz force vanishes. In MHD this assumption translates into a specific class of magnetic field configurations. However, even in the case of a force-free configuration the specific initial conditions used by the authors in order to compute the CMB anisotropies are not specifically discussed, i.e., no solution for superhorizon sized fluctuations including large-scale magnetic fields is presented. Furthermore, no specific investigation of the impact of gravitating magnetic fields on the tightcoupling expansion has been attempted. In Ref. [9] an interesting analysis of the impact of large-scale magnetic fields on CMB anisotropies has been performed, mainly for the vector and tensor modes of the geometry. The scalar fluctuations have not been discussed. In Ref. [10] attention is always paid to vector and tensor modes and the problem of initial conditions is more accurately specified. It is clear that without a precise specification of the initial conditions for the CMB anisotropies the numerical analysis is rather difficult to implement since it is unclear what should be, for instance, the initial values of the lowest multipoles of the phase-space distribution for the various species. The need of an accurate analysis of the problem of initial conditions can be also understood by observing that the system of perturbed equations, even if linear, has many unknowns so different solutions describing a different physical situation may be possible.

The effect of large-scale magnetic fields on CMB anisotropies may also be discussed in the case when the magnetic field is not completely inhomogeneous. There could be situations in which there is an homogeneous component of the magnetic field (for instance along the $\hat{z}$ axis). This case is similar to the one originally investigated in Refs. [1,2]. In this framework bounds on such a magnetic field can be derived [11]. It could also happen that the uniform magnetic field supports inhomogeneities in the bulk velocity field affecting, consequently, the CMB anisotropies. One example in this direction are the Alfvén waves, whose effects and implications have been analyzed under different approximations by various authors [12-15]. The simplification of having a uniform magnetic field also allowed the analysis of possible Faraday rotation effect of the CMB polarization plane [16-19].

\footnotetext{
${ }^{1}$ By standard CMB physics we mean that a constant mode of adiabatic (or quasiadiabatic) fluctuations is assumed to be present outside the horizon prior to equality.
} 
The motivation for including a uniform magnetic field relies on the simplicity of the configuration. However, the magnetic fields produced in the early Universe, as far as we understand the problem, are unlikely to be produced in a perfectly uniform configuration. They are most likely to be produced either from the amplification of vacuum fluctuations of some primordial gauge field or from some phase transitions. In all these cases magnetic fields are expected to be fully inhomogeneous (see, for instance, [20] for a recent review on the role of large-scale magnetic fields in cosmology and astrophysics).

The purpose of the present study is to give a systematic analysis of the theory of scalar gravitational fluctuations in the presence of a fully inhomogeneous magnetic field and in the context of the conventional Bardeen formalism. This analysis is mandatory both to derive accurate bounds on large-scale magnetic fields from CMB physics and to bring the study of magnetized initial conditions to the same standard as in the nonmagnetized case. To the best of our knowledge this problem did not receive specific attention in the literature concerning the linearized theory of scalar fluctuations in the presence of magnetic fields. The results reported in the present paper are general in the sense that no specific configuration of the magnetic field is assumed. The only assumption, as previously stressed, is that the magnetic field is fully inhomogeneous.

It is important to mention that various studies discussing the evolution of large-scale magnetic field exist in the covariant approach which is somehow complementary to the one adopted in the present paper. For a full discussion of the problem see [21,22] and references therein.

The plan of the present paper is the following. In Sec. II the evolution equations for gravitating inhomogeneous magnetic fields will be derived in the MHD approximation and in the context of the conformally Newtonian gauge. In Sec. III the initial conditions for the CMB anisotropies will be analyzed. Solutions for superhorizon-sized fluctuations will be presented in different cases. Section IV is devoted to the analysis of the synchronous gauge description. In Sec. $\mathrm{V}$ the tightcoupling expansion will be revisited in the presence of inhomogeneous magnetic fields. Section VI contains some concluding remarks. Various useful technical results have been collected in the appendix even though, in some cases, they were already present in the literature, but within a different set of conventions or within a different context.

\section{GRAVITATING INHOMOGENEOUS MAGNETIC FIELDS}

The treatment of magnetic fields in a globally neutral plasma differs considerably according to the specific range of scales relevant for the problem under discussion.
For instance, the electromagnetic branch of the spectrum of plasma excitations, in a cold or warm magnetized plasma, can only be partially addressed within a twofluid description [23,24]. Partially means, in the present context, that the dispersion relations can be obtained but the typical damping scales require the solution of the full kinetic system of equations for the different species (the so-called Vlasov-Landau approach).

The phenomena occurring over much smaller frequencies and over large length scales can be described on the basis of our knowledge of terrestrial plasma, by an appropriate one-fluid description, which is provided by MHD [25]. The main idea behind the MHD approximation is, in short, the following. Starting from a two-fluid description (for instance electron and baryon fluids) it is possible to define appropriate one-fluid variables. For instance the bulk velocity of the plasma appearing in the MHD description is, in flat-space-time,

$$
\vec{v}=\frac{m_{\mathrm{b}} \overrightarrow{\boldsymbol{v}}_{\mathrm{b}}+m_{\mathrm{e}} \vec{v}_{\mathrm{e}},}{m_{\mathrm{b}}+m_{\mathrm{e}}},
$$

which is, in practice, the center-of-mass velocity of the baryon-electron system. In the specific case touched by the present discussion, both baryons and electrons are nonrelativistic and in thermal equilibrium $T_{\mathrm{eb}}$.

The reduced MHD description [25] has been employed in the analysis of different problems arising in connection with large-scale magnetic fields. For instance, the evolution of these fields in curved space-times is normally discussed within a MHD approach [26-28] (see also for a review [29,30]). More formal discussions on MHD and on two-fluid descriptions in curved space-times can be found in Refs. [31-33]. Finally, the reduced MHD description has been used in the analysis of the implications of large-scale magnetic fields on structure formation [34,35].

\section{A. The perturbed system of Einstein equations}

Consider now the Friedmann-Robertson-Walker (FRW) line element written in the conformally flat case

$$
d s^{2}=a^{2}(\eta)\left[d \eta^{2}-d \vec{x}^{2}\right],
$$

where $\eta$ is the conformal time coordinate. Since the magnetic fields are fully inhomogeneous, they will not affect the homogeneous background whose equations are, in the spatially flat case,

$$
\mathcal{H}^{2}=\frac{8 \pi G}{3} a^{2} \rho \sum_{\lambda} \Omega_{\lambda},
$$

$$
\begin{gathered}
\mathcal{H}^{2}-\mathcal{H}^{\prime}=4 \pi G a^{2} \rho \sum_{\lambda} \Omega_{\lambda}\left(1+w_{\lambda}\right), \\
\rho_{\lambda}^{\prime}+3 \mathcal{H}\left(\rho_{\lambda}+p_{\lambda}\right)=0,
\end{gathered}
$$


where $\mathcal{H}=(\ln a)^{\prime}$ and the prime denotes a derivation with respect to $\eta$. In Eqs. (2.3), (2.4), and (2.5) the summation index $\lambda$ refers to each component of the plasma, i.e., baryons, photons, neutrinos and CDM particles; $w_{\lambda}=p_{\lambda} / \rho_{\lambda}$ and $\Omega_{\lambda}$ are, respectively, the barotropic index for the given species $\lambda$ and the energy density (in critical units) for each component of the fluid. In view of the subsequent discussions, it is appropriate to define here also the usual Hubble parameter, i.e., $H=\dot{a} / a$, where the overdot denotes a derivation with respect to the cosmic time coordinate $t$, which is related to $\eta$ by the differential relation $d \eta a(\eta)=d t$. In terms of these definitions we also have $\mathcal{H}=a H$.

Let us now consider the fluctuations of the homogeneous FRW metric (2.2). In this section the conformally Newtonian coordinate system will be discussed and, therefore, the metric (2.2) can be consistently perturbed in terms of the two longitudinal scalar degrees of freedom $\phi$ and $\psi$

$$
\delta g_{00}=2 a^{2} \phi, \quad \delta g_{i j}=2 a^{2} \psi \delta_{i j} .
$$

Defining the fluctuations in the Ricci tensor and in the Ricci scalar as $\delta R_{\mu}^{\nu}$ and $\delta R$, the perturbed Einstein equations,

$$
\delta R_{\mu}^{\nu}-\frac{1}{2} \delta_{\mu}^{\nu} \delta R=8 \pi G a^{2}\left(\delta T_{\mu}^{\nu}+\delta \tau_{\mu}^{\nu}\right),
$$

relate the fluctuations of the geometry to the fluctuations of the matter sources, $\delta T_{\mu}^{\nu}$, and to the fluctuations of the electromagnetic energy-momentum tensor, $\delta \tau_{\mu}^{\nu}$.

In explicit terms the (00), $(0 i)$ and $(i j)$ components of Eq. (2.7) lead, respectively, to

$$
\begin{gathered}
\nabla^{2} \psi-3 \mathcal{H}\left(\mathcal{H} \phi+\psi^{\prime}\right)=4 \pi G a^{2}\left[\delta T_{0}^{0}+\delta \tau_{0}^{0}\right], \\
-\partial^{i}\left(\mathcal{H} \phi+\psi^{\prime}\right)=4 \pi G a^{2}\left(\delta T_{0}^{i}+\delta \tau_{0}^{i}\right), \\
{\left[\psi^{\prime \prime}+\mathcal{H}\left(2 \psi^{\prime}+\phi^{\prime}\right)+\left(2 \mathcal{H}^{\prime}+\mathcal{H}^{2}\right) \phi+\frac{1}{2} \nabla^{2}(\phi\right.} \\
-\psi)] \delta_{i}^{j}-\frac{1}{2} \partial_{i} \partial^{j}(\phi-\psi) \\
=-4 \pi G a^{2}\left[\delta T_{i}^{j}+\delta \tau_{i}^{j}\right],
\end{gathered}
$$

where

$$
\begin{gathered}
\delta \tau_{0}^{0}=\frac{1}{8 \pi a^{4}}\left(\vec{E}^{2}+\vec{B}^{2}\right), \\
\delta \tau_{0}^{i}=\frac{1}{4 \pi a^{4}} \vec{E} \times \vec{B}, \\
\delta \tau_{i}^{j}=\frac{1}{4 \pi a^{4}}\left[E_{i} E^{j}+B_{i} B^{j}-\frac{1}{2}\left(\vec{B}^{2}+\vec{E}^{2}\right) \delta_{i}^{j}\right], \\
\delta T_{0}^{0}=\sum_{\lambda} \rho_{\lambda} \delta_{\lambda},
\end{gathered}
$$

$$
\begin{gathered}
\delta T_{i}^{j}=-\sum_{\lambda} w_{\lambda} \rho_{\lambda} \delta_{\lambda} \delta_{i}^{j}+\Sigma_{i}^{j}, \\
\delta T_{0}^{i}=\sum_{\lambda}\left(1+w_{\lambda}\right) \rho_{\lambda} v_{\lambda}^{i},
\end{gathered}
$$

where $\delta_{\lambda}=\delta \rho_{\lambda} / \rho_{\lambda}$ and $v_{\lambda}^{i}$ are, respectively, the density contrast and the peculiar velocity for each particle species; $\Sigma_{i}^{j}$ is the traceless component of the energymomentum tensor of the fluid sources, i.e.,

$$
\Sigma_{i}^{j}=\delta T_{i}^{j}-\frac{1}{3} \delta_{i}^{j} \delta T .
$$

According to the usual notation the anisotropic stress can also be written, in Fourier space, as

$$
\mathcal{Q}=\partial_{j} \partial^{i} \Sigma_{i}^{j}=-k^{2} \sum_{\lambda} \sigma_{\lambda}\left(1+w_{\lambda}\right) \rho_{\lambda},
$$

where $\sigma_{\lambda}$ denotes the fraction contributed by each species $\lambda$ to the total anisotropic stress. Notice, as it will be more extensively discussed later, that the dominant source of anisotropic stress comes, for temperatures below the $\mathrm{MeV}$, from the neutrinos.

Finally in Eqs. (2.11), (2.12), and (2.13) the components of the energy-momentum tensor have been written directly using the rescaled electric and magnetic fields $\vec{E}$ and $\vec{B}$, which can also be expressed in terms of the corresponding flat-space fields as

$$
\vec{E}(\eta, \vec{x})=a^{2}(\eta) \overrightarrow{\mathcal{E}}(\eta, \vec{x}), \quad \vec{B}(\eta, \vec{x})=a^{2}(\eta) \overrightarrow{\mathcal{B}}(\eta, \vec{x}) .
$$

In the following the evolution of the different quantities appearing in the right-hand side of Einstein equations will be discussed in detail.

\section{B. Electromagnetic fields and baryons}

The Maxwell equations can be studied using the rescaled fields proposed in Eqs. (2.19)

$$
\begin{gathered}
\vec{B}^{\prime}=-\vec{\nabla} \times \vec{E}, \\
\vec{\nabla} \times \vec{B}=4 \pi \vec{J}+\vec{E}^{\prime}, \\
\vec{\nabla} \cdot \vec{E}=4 \pi \rho_{\mathrm{q}}, \\
\vec{\nabla} \cdot \vec{B}=0,
\end{gathered}
$$

where $\rho_{\mathrm{q}}$ is the charge density $\left(\rho_{\mathrm{q}} \simeq 0\right.$ in a globally neutral plasma); $\vec{J}=a^{3} \vec{j}$ is the Ohmic current density

$$
\vec{J}=\sigma(\vec{E}+\vec{v} \times \vec{B}) .
$$

In Eq. (2.24), $\sigma$ is the conductivity that is related to the flat-space conductivity as $\sigma=a \sigma_{c}$. Notice that in the reduced MHD description the Ohm law follows, in the reduced MHD approach, by taking the difference of 
the equations describing the momentum conservation for electrons and for ions. This procedure is rather tricky and, in principle other terms may appear in the generalized Ohm law such as the thermoelectric term and the Hall term [20,23]. These will be neglected here. Finally, as pointed out after Eq. (2.1), the bulk velocity field appearing in Eq. (2.24) can be identified with the velocity of the baryons.

The evolution for the baryon velocity field can be derived by perturbing the covariant conservation equation (including the appropriate electromagnetic contribution), i.e.,

$$
\begin{aligned}
\partial_{\mu} \delta T_{\mathrm{b}}^{\mu \nu} & +\delta \Gamma_{\mu \alpha}^{\nu} \bar{T}_{\mathrm{b}}^{\mu \alpha}+\bar{\Gamma}_{\mu \alpha}^{\nu} \delta T_{\mathrm{b}}^{\mu \alpha}+\delta \Gamma_{\beta \mu}^{\mu} \bar{T}_{\mathrm{b}}^{\beta \nu} \\
& +\bar{\Gamma}_{\beta \mu}^{\mu} \delta T_{\mathrm{b}}^{\beta \nu}-F^{\nu \alpha} j_{\alpha}=0,
\end{aligned}
$$

where $\bar{T}_{\mathrm{b}}^{\mu \nu}$ and $\delta T_{\mathrm{b}}^{\mu \nu}$ are, respectively, the energy momentum of the baryons and its first-order fluctuation; $F^{\nu \alpha}$ is the Maxwell field strength and $j_{\alpha}$ is the current density. An analogous notation is used for the Christoffel symbols computed on the background (denoted by an overline) and for their first-order fluctuations. Thus, defining $\theta_{\mathrm{b}}=\vec{\nabla}$. $\vec{v}_{\mathrm{b}}$, the evolution equation for the peculiar velocity of the baryons and for the density contrast reads:

$$
\begin{gathered}
\theta_{\mathrm{b}}^{\prime}=-\mathcal{H} \theta_{\mathrm{b}}-c_{s}^{2} \nabla^{2} \delta_{\mathrm{b}}-\nabla^{2} \phi+\frac{4}{3} \frac{\Omega_{\gamma}}{\Omega_{\mathrm{b}}} a n_{\mathrm{e}} x_{\mathrm{e}} \sigma_{\mathrm{T}}\left(\theta_{\gamma}-\theta_{\mathrm{b}}\right) \\
+\frac{\vec{\nabla} \cdot[\vec{J} \times \vec{B}]}{a^{4} \rho_{b}}, \\
\delta_{\mathrm{b}}^{\prime}=3 \psi^{\prime}-\theta_{\mathrm{b}},
\end{gathered}
$$

where $x_{\mathrm{e}}$ is the ionization fraction of the plasma, $\sigma_{\mathrm{T}}$ the Thompson cross section; $\Omega_{\gamma} \Omega_{\mathrm{b}}$ are, respectively, the energy densities, in critical units, for photons and baryons. At this point we can also define the differential optical depth for Thompson scattering:

$$
\tau^{\prime}=x_{\mathrm{e}} n_{\mathrm{e}} \sigma_{\mathrm{T}} a(\eta) .
$$

Equations (2.20), (2.21), (2.22), and (2.23), together with Eqs. (2.24) and (2.26), can be studied in the MHD approximation. Since the plasma is globally neutral $\left(\rho_{\mathrm{q}} \simeq\right.$ $0)$, the charge density of the electrons will be exactly compensated by the baryons and, according to Eq. (2.22), the electric field will be solenoidal. Moreover, for sufficiently small frequencies, the displacement current can be neglected in Eq. (2.21). This implies that also the Ohmic current will be solenoidal, i.e.,

$$
\vec{\nabla} \cdot \vec{J}=0, \quad \vec{J}=\frac{1}{4 \pi} \vec{\nabla} \times \vec{B} .
$$

As a result, the Ohmic electric field vanishes exactly in the limit of vanishing conductivity, i.e., since

$$
\vec{E} \sim \frac{\vec{J}}{\sigma}=\frac{\vec{\nabla} \times \vec{B}}{\sigma},
$$

the electric fields appearing in Eqs. (2.11) and (2.13) lead to a contribution that is suppressed as $1 / \sigma^{2}$. Similarly, the electric field appearing in Eq. (2.12) leads to a contribution that is suppressed as $1 / \sigma$.

Recalling, as previously defined, that $\sigma=\sigma_{c} a$, for temperatures $T<1 \mathrm{MeV}$ the conductivity can be expressed as $[20,23]$

$$
\sigma_{c} \simeq \frac{1}{\alpha_{\mathrm{em}}}\left(\frac{T_{\mathrm{eb}}}{m_{\mathrm{e}}}\right)^{1 / 2},
$$

where $T_{\mathrm{eb}}$, as previously defined, is the common temperature of electrons and baryons. The finite value of the conductivity sets a typical diffusion scale for the evolution of the magnetic fields which is typically of the order of

$$
L_{\sigma}(T)=\frac{\sigma_{0}^{-1 / 2} g_{*}^{1 / 4}}{72.2}\left(\frac{T_{\mathrm{eb}}}{M_{\mathrm{P}}}\right)^{1 / 2}\left(\frac{T_{\mathrm{eb}}}{m_{\mathrm{e}}}\right)^{-1 / 4} L_{H}(T),
$$

where $L_{\sigma}$ is the physical diffusion scale, $L_{H} \simeq H^{-1}$ is the Hubble radius and $g_{*}$ the number of relativistic degrees of freedom. Since, in our problem eV $<T_{\text {eb }}<\mathrm{MeV}, L_{\sigma} \ll$ $L_{H}$ which means that the finite value of the conductivity only affects scales which are much smaller than the Hubble radius.

Similar, but quantitatively different, remarks can be made for the thermal diffusivity [20]. For temperature smaller than the temperature of neutrino decoupling, photons are the most efficient source of momentum transfer and the thermal diffusivity scale can be written as

$$
\begin{aligned}
\frac{L_{\text {diff }}^{(\gamma)}(T)}{L_{H}(T)} \simeq & 1.03 \times 10^{-5}\left(\frac{\Omega_{\mathrm{b}} h_{0}^{2}}{0.02}\right)^{-1 / 2}\left(\frac{x_{\mathrm{e}}}{0.5}\right)^{-1 / 2}\left(\frac{g_{*}}{10.75}\right)^{1 / 4} \\
& \times \sqrt{\frac{\mathrm{MeV}}{T_{\mathrm{eb}}}},
\end{aligned}
$$

where, according to previous definitions [see Eq. (2.26)], $x_{\mathrm{e}}$ is the ionization fraction, $\Omega_{\mathrm{b}}$ the energy density of baryons in critical units.

\section{Cold dark matter}

The evolution of the CDM particles follows from the fluctuations of the covariant conservation equation whose first-order fluctuation leads, in Fourier space, to

$$
\begin{gathered}
\theta_{\mathrm{c}}^{\prime}+\mathcal{H} \theta_{c}=k^{2} \phi, \\
\delta_{\mathrm{c}}^{\prime}=3 \psi^{\prime}-\theta_{\mathrm{c}},
\end{gathered}
$$

where, following the previous notation, $\theta_{\mathrm{c}}=\partial_{i} v_{\mathrm{c}}^{i}$ and $\delta_{\mathrm{c}}=\delta \rho_{\mathrm{c}} / \rho_{\mathrm{c}}$. 


\section{Photons and massless neutrinos}

For the neutrinos the evolution equations follow only partially from the energy-momentum conservation. In fact neutrinos at early times after neutrino decoupling (occurring around $1 \mathrm{MeV}$ ) obey the collisionless Boltzmann equation (see the appendix for a more detailed discussion). To describe neutrinos during and after horizon crossing requires a Boltzmann hierarchy for $\delta_{\nu}, \theta_{\nu}$ and for the higher multipole moments, i.e., $\ell \geq 2$, of the neutrino phase-space density $\mathcal{F}_{\nu \ell}$. With this caveat in mind, after neutrino decoupling, at temperatures of about $1 \mathrm{MeV}$, massless neutrinos obey, in Fourier space, the following set of equations [see the appendix for a swift derivation within the metric conventions adopted in Eqs. (2.2) and (2.6)]:

$$
\begin{gathered}
\delta_{\nu}^{\prime}=-\frac{4}{3} \theta_{\nu}+4 \psi^{\prime}, \\
\theta_{\nu}^{\prime}=\frac{k^{2}}{4} \delta_{\nu}-k^{2} \sigma_{\nu}+k^{2} \phi, \\
\sigma_{\nu}^{\prime}=\frac{4}{15} \theta_{\nu}-\frac{3}{10} k \mathcal{F}_{\nu 3},
\end{gathered}
$$

where $\sigma_{\nu}=\mathcal{F}_{\nu 2} / 2$ is the quadrupole moment of the (perturbed) neutrino phase-space distribution and, as introduced above, $\mathcal{F}_{\nu \ell}$ is the $\ell$ th multipole.

The photon and neutrino evolution equations differ in the presence of an anisotropic stress term $\sigma_{\nu}$. Because of their frequent scattering by charged leptons and baryons, photons are unable, at early times, to develop a quadrupole moment in their velocity distribution. As a consequence, when a mode enters the horizon, the photons behave as a perfect fluid, while the neutrinos free stream, creating inhomogeneities in the energy density, pressure and momentum density. This is the physical reason why the anisotropic stress term appearing in Eq. (2.15) is effectively dominated, at early times, by the neutrino contribution. Also for the photons it is more appropriate to discuss the full Boltzmann hierarchy, especially in light of the subsequent analysis of the tight-coupling approximation. This analysis will be summarized in the appendix. In the fluid approximation the evolution equations of the photons are given by the covariant conservation of the energy-momentum tensor, i.e.,

$$
\begin{gathered}
\delta_{\gamma}^{\prime}=-\frac{4}{3} \theta_{\gamma}+4 \psi^{\prime}, \\
\theta_{\gamma}^{\prime}=\frac{k^{2}}{4} \delta_{\gamma}+k^{2} \phi+a n_{\mathrm{e}} \sigma_{\mathrm{T}}\left(\theta_{\mathrm{b}}-\theta_{\gamma}\right) .
\end{gathered}
$$

At early times the characteristic time for the synchronization of the photon and baryon velocities is $\tau_{\mathrm{b} \gamma} \sim$ $\left(n_{\mathrm{e}} \sigma_{\mathrm{T}}\right)^{-1}$, which is small with respect to the expansion time and to the oscillation period. Thus the tight coupling between photons and baryons means that, in the first approximation, $\sigma_{\mathrm{T}} \rightarrow \infty$ and $\theta_{\gamma} \simeq \theta_{\mathrm{b}}$. A more detailed analysis of the tight-coupling expansion is postponed to Sec. V.

\section{LARGE-SCALE SOLUTIONS}

The full system of scalar fluctuations in the longitudinal gauge will now be consistently discussed and solved. From Eq. (2.8) we obtain, in Fourier space ${ }^{2}$ and using Eq. (2.3):

$$
\begin{aligned}
-3 \mathcal{H}\left(\mathcal{H} \phi+\psi^{\prime}\right)-k^{2} \psi= & \frac{3}{2} \mathcal{H}^{2}\left\{\left[R_{\nu} \delta_{\nu}+\left(1-R_{\nu}\right) \delta_{\gamma}\right]\right. \\
& \left.+\Omega_{\mathrm{B}}(k)+\Omega_{\mathrm{b}} \delta_{\mathrm{b}}+\Omega_{\mathrm{c}} \delta_{\mathrm{c}}\right\},
\end{aligned}
$$

where, for $N_{\nu}$ species of massless neutrinos,

$$
R=\frac{7}{8} N_{\nu}\left(\frac{4}{11}\right)^{4 / 3}, \quad R_{\nu}=\frac{R}{1+R}, \quad R_{\gamma}=1-R_{\nu},
$$

so that $R_{\nu}$ and $R_{\gamma}$ represent the fractional contributions of photons and neutrinos to the total density at early times deep within the radiation-dominated epoch.

In Eq. (3.1) the contribution of the magnetic energy density has been parametrized, in Fourier space, as

$$
\Omega_{\mathrm{B}}(k, \eta)=\frac{\rho_{\mathrm{B}}}{\rho}=\frac{1}{8 \pi \rho a^{4}} \int d^{3} p B_{i}(|\vec{p}-\vec{k}|) B^{i}(p) .
$$

The appearance of convolutions in the expressions of the magnetic energy density is a direct consequence of the absence of a uniform component of the magnetic field whose background contribution, as repeatedly stressed, is vanishing. Notice also that $\rho$ appearing in Eq. (3.3) is the energy density of the dominant component of the fluid, so, for instance, in the radiation-dominated epoch $\rho \equiv$ $\rho_{\mathrm{r}}, a^{4} \rho_{\mathrm{r}} \sim$ const and $\Omega_{B} \simeq$ const.

From Eq. (2.9), using the same notation as in Eq. (3.1), the following simplified equation can be obtained for the momentum constraint:

$$
\begin{aligned}
k^{2}\left(\mathcal{H} \phi+\psi^{\prime}\right)= & \frac{3}{2} \mathcal{H}^{2}\left\{\frac{4}{3}\left[R_{\nu} \theta_{\nu}+\left(1-R_{\nu}\right) \theta_{\gamma}\right]\right. \\
& \left.+\frac{F_{\mathrm{B}}(k)}{4 \pi \sigma a^{4} \rho}+\theta_{\mathrm{b}} \Omega_{\mathrm{b}}+\theta_{\mathrm{c}} \Omega_{\mathrm{c}}\right\},
\end{aligned}
$$

where $F_{\mathrm{B}}(k, \eta)$ is the Fourier transform of the generalized Lorentz force, i.e.,

$$
\vec{\nabla} \cdot[(\vec{\nabla} \times \vec{B}) \times \vec{B}]=\int d^{3} k F_{\mathrm{B}}(k) e^{i \vec{k} \cdot \vec{x}},
$$

where $F_{\mathrm{B}}(k)$ is given by the following convolution:

\footnotetext{
${ }^{2}$ In order to avoid possible confusions with subscripts referring to the different species of the plasma we will avoid introducing a further subscript labeling the Fourier mode.
} 


$$
\begin{aligned}
& \int d^{3} p\left[(\vec{k} \cdot \vec{p}) B_{j}(p) B^{j}(|\vec{k}-\vec{p}|)-\left(k_{i}-p_{i}\right) B_{j}(\mid \vec{k}\right. \\
& \left.-\vec{p} \mid) B_{j}(|\vec{k}-\vec{p}|) B^{j}(p)\right] .
\end{aligned}
$$

Notice that a consistent treatment of fully inhomogeneous magnetic fields implies that

$$
\Omega_{\mathrm{B}} \ll 1, \quad \frac{F_{\mathrm{B}}}{4 \pi k^{2} \rho a^{4}} \ll 1,
$$

in order not to overclose the Universe.

In the ideal MHD limit ${ }^{3}$, i.e., $\sigma \rightarrow \infty$, the contribution of $F_{\mathrm{B}}(k)$ disappears from Eq. (3.4). This occurrence is not general: it will be shown in a moment that, even in the ideal MHD limit, the generalized Lorentz force does contribute both to the baryon evolution equation and to the anisotropic stress. In fact the evolution equation for the baryons becomes, in Fourier space and with the conventions used so far,

$$
\theta_{\mathrm{b}}^{\prime}+\mathcal{H} \theta_{\mathrm{b}}=k^{2} \phi+\frac{F_{\mathrm{B}}(k)}{4 \pi a^{4} \rho_{\mathrm{b}}} .
$$

By taking the trace of Eq. (2.10) it is easy to obtain

$$
\begin{aligned}
\psi^{\prime \prime} & +\left(2 \psi^{\prime}+\phi^{\prime}\right) \mathcal{H}+\left(2 \mathcal{H}^{\prime}+\mathcal{H}^{2}\right) \phi-\frac{k^{2}}{3}(\phi-\psi) \\
& =\frac{1}{2} \mathcal{H}^{2}\left\{\left[R_{\nu} \delta_{\nu}+\left(1-R_{\nu}\right)\right] \delta_{\gamma}+\Omega_{\mathrm{B}}(k)\right\},
\end{aligned}
$$

where the contribution of the other species vanishes since both baryons and CDM particles have a vanishing barotropic index, i.e., $w_{\mathrm{b}}=w_{\mathrm{c}}=0$.

By taking now the difference between Eqs. (2.10) and (3.9), we can get the equation for the anisotropic stress, i.e., for the perturbed components $i \neq j$ of the Einstein equations:

$$
\begin{aligned}
\partial_{i} \partial^{j}(\phi-\psi)-\frac{1}{3} \nabla^{2}(\phi-\psi)= & 8 \pi G a^{2} \rho\left\{\Sigma_{i}^{j}+\frac{1}{4 \pi \rho a^{4}}\right. \\
& \left.\times\left[B_{i} B^{j}-\frac{1}{3} \vec{B}^{2} \delta_{i}^{j}\right]\right\} .
\end{aligned}
$$

Applying now the differential operator $\partial_{j} \partial^{i}$ to Eq. (3.10), the following relation can be obtained:

$\nabla^{4}(\phi-\psi)=12 \pi G a^{2}\left[\mathcal{Q}+\frac{1}{4 \pi a^{4} \rho}\left(\partial_{i} B_{j} \partial^{j} B^{i}-\frac{1}{3} \nabla^{2} \vec{B}^{2}\right)\right]$

\footnotetext{
${ }^{3}$ Notice that, so far, different quantities have been denoted by $\sigma$ (with different indices), namely, the Thompson cross section (i.e., $\sigma_{\mathrm{T}}$ ), the conductivity (i.e., $\sigma$ ) and the fractional contribution of each species $\lambda$ to the anisotropic stress (i.e., $\sigma_{\lambda}$ ). Since all the quantities are properly defined and used, there should be no confusion. We felt that it would be even more confusing if we changed the usually accepted notations for these three important quantities.
}

where, following the conventions of Eq. (2.18),

$$
\mathcal{Q}=\partial_{i} \partial^{j} \sum_{j}^{i}=-k^{2} \sigma_{\nu}\left(p_{\nu}+\rho_{\nu}\right)=-\frac{4}{3} k^{2} \rho_{\nu} .
$$

In order to write Eq. (3.12) in an explicit form it has been assumed that the only anisotropic stress arising from the fluid sources is the one related to the quadrupole moment of the neutrino phase-space density. Going to Fourier space, Eq. (3.11) becomes

$$
k^{2}(\phi-\psi)=\frac{9}{2} \mathcal{H}^{2}\left[-\frac{4}{3} \sigma_{\nu}-\sigma_{\mathrm{B}}(k)\right],
$$

having defined

$$
\sigma_{\mathrm{B}}=\frac{\Omega_{\mathrm{B}}(k)}{3}-\frac{F_{\mathrm{B}}(k)}{4 \pi a^{4} \rho k^{2}} .
$$

The quantity $\sigma_{\mathrm{B}}$ can be interpreted as the anisotropic stress arising thanks to the inhomogeneous magnetic field and, in the force-free case, $\sigma_{\mathrm{B}}=\Omega_{\mathrm{B}} / 3$.

In order to discuss the initial conditions for the CMB anisotropies, the usual procedure is to solve the system of equations for the fluctuations while the Fourier modes of the various fields are outside the horizon, i.e., $k \eta<1$. Different sorts of initial conditions are, in principle, possible. An interesting situation arises when both $\psi$ and $\phi$ are, in the first approximation, constant outside the horizon during the radiation-dominated epoch. In this case, and in the absence of magnetic fields, it is known that the system of scalar metric perturbations admits an adiabatic mode. Adiabatic means, in the present context, that the total fluctuations in the entropy density of the CDM-baryon-radiation fluid vanish at large length scales. This condition implies specific relations between the various density contrasts deep within the radiationdominated epoch, i.e.,

$$
\delta_{\mathrm{c}} \simeq \delta_{\mathrm{b}} \simeq \frac{3}{4} \delta_{\nu} \simeq \frac{3}{4} \delta_{\gamma} .
$$

In the following, after reviewing the specific analytic form of the adiabatic solution, new solutions, arising in the presence of an inhomogeneous magnetic field, will be illustrated.

\section{A. The standard adiabatic scenario}

If the magnetic fields are absent, i.e., $F_{\mathrm{B}}=\Omega_{B}=0$ the standard system of equations is recovered and the initial conditions for the various fields can be found by solving, simultaneously, the baryon-photon system [i.e., Eqs. (2.26), (2.27), (2.39), and (2.40)], the CDM and neutrinos equations [i.e., Eqs. (2.34), (2.35), (2.36), (2.37), and (2.38)] together with the perturbed Einstein equations whose specific form has been derived above from Eq. (3.1) to Eq. (3.13). In the following, only the final result will be given: 


$$
\begin{gathered}
\bar{\delta}_{\mathrm{b}}=\bar{\delta}_{\mathrm{c}}=-\frac{3}{2} \phi_{0}-\frac{\left(525+188 R_{\nu}+16 R_{\nu}^{2}\right)}{60\left(25+2 R_{\nu}\right)} \phi_{0} k^{2} \eta^{2} \\
\bar{\delta}_{\gamma}=\bar{\delta}_{\nu}=-2 \phi_{0}-\frac{\left(525+188 R_{\nu}+16 R_{\nu}^{2}\right)}{45\left(25+2 R_{\nu}\right)} \phi_{0} k^{2} \eta^{2} \\
\bar{\phi}=\phi_{0}-\frac{\left(75+14 R_{\nu}-8 R_{\nu}^{2}\right)}{90\left(25+2 R_{\nu}\right)} \phi_{0} k^{2} \eta^{2} \\
\bar{\psi}^{2}=\left(1+\frac{2}{5} R_{\nu}\right) \phi_{0}-\frac{\left(75+79 R_{\nu}+8 R_{\nu}^{2}\right)}{90\left(25+2 R_{\nu}\right)} \phi_{0} k^{2} \eta^{2} \\
\bar{\theta}_{\nu}=\frac{\phi_{0}}{2} k^{2} \eta-\frac{\left(65+16 R_{\nu}\right)}{36\left(25+2 R_{\nu}\right)} \phi_{0} k^{4} \eta^{3} \\
\bar{\theta}_{\mathrm{b}}=\frac{\phi_{0}}{2} k^{2} \eta-\frac{\left(75+14 R_{\nu}-8 R_{\nu}^{2}\right)}{360\left(25+2 R_{\nu}\right)} \phi_{0} k^{4} \eta^{3} \\
\bar{\theta}_{\mathrm{c}}=\frac{\phi_{0}}{2} k^{2} \eta-\frac{\left(75+14 R_{\nu}-8 R_{\nu}^{2}\right)}{360\left(25+2 R_{\nu}\right)} \phi_{0} k^{4} \eta^{3} \\
\bar{\theta}_{\gamma}=\frac{\phi_{0}}{2} k^{2} \eta-\frac{\left(25+8 R_{\nu}\right)}{20\left(25+2 R_{\nu}\right)} \phi_{0} k^{2} \eta^{2} \\
\bar{\sigma}^{2} \eta^{2}-\frac{\left(65+16 R_{\nu}\right)}{540\left(25+2 R_{\nu}\right)} \phi_{0} k^{4} \eta^{4}
\end{gathered}
$$

where the overline in the various quantities has been introduced for future notational convenience. Concerning this solution, a few comments are in order:

(i) while the leading order of the solution is usually quoted (see for instance [5,36]), the second is useful in the present case, in order to check what happens at the horizon crossing of a given mode (i.e. $k \eta \sim 1$ ) and in order to compare with the situation where the magnetic field is present;

(ii) while to leading order all the peculiar velocities of the plasma coincide, to next-to-leading order they are different;

(iii) the adiabaticity condition given in Eq. (3.15) holds not only to leading order but also to next-toleading order in $|k \eta|^{2}$.

\section{B. Force-free case}

Consider now the case when the magnetic field is forcefree, and suppose, as previously discussed, that the solution at large scale is, in the first approximation, a constant mode for the longitudinal fluctuations of the metric. Then, the solution can be parametrized as

$$
\phi \simeq \phi_{0}+C_{\phi} k^{2} \eta^{2}, \quad \psi \simeq \psi_{0}+C_{\psi} k^{2} \eta^{2} .
$$

We are interested in the solution of the system deep in the radiation epoch where $a(\eta) \sim \eta$ and the dominant component of the fluid energy-momentum tensor consist of photons and neutrinos, i.e., $\Omega_{\mathrm{b}} \ll 1$ and $\Omega_{\mathrm{c}} \ll 1$. From Eq. (3.1), the density contrasts for photons and neutrinos can be written as

$$
\begin{gathered}
\delta_{\gamma}=-2 \phi_{0}-\Omega_{\mathrm{B}}+A_{\gamma} k^{2} \eta^{2}, \\
\delta_{\nu}=-2 \phi_{0}-\Omega_{\mathrm{B}}+A_{\nu} k^{2} \eta^{2},
\end{gathered}
$$

where the constants $A_{\gamma}$ and $A_{\nu}$ are related to $C_{\phi}$ and $C_{\psi}$ by the following algebraic equation:

$$
C_{\phi}+2 C_{\psi}+\frac{\psi_{0}}{3}=-\frac{1}{2}\left[R_{\nu} A_{\nu}+\left(1-R_{\nu}\right) A_{\gamma}\right] .
$$

Inserting Eqs. (3.25) together with Eq. (3.26) into Eqs. (2.39) and (2.40), the following parametrization for the peculiar velocity of the photons can be derived:

$$
\theta_{\gamma}=k^{2}\left[\frac{\phi_{0}}{2}-\frac{\Omega_{\mathrm{B}}}{4}\right] \eta+D_{\gamma} k^{4} \eta^{3}
$$

together with two algebraic relations determining the constants

$$
\begin{gathered}
3 D_{\gamma}=\frac{A_{\gamma}}{4}+C_{\phi}, \\
A_{\gamma}=-\frac{\phi_{0}}{3}+\frac{\Omega_{\mathrm{B}}}{6}+4 C_{\psi} .
\end{gathered}
$$

With a similar procedure the baryon and CDM fluids can be analyzed: from Eqs. (2.26), (2.27), (2.34), and (2.35), the solution can be written as follows:

$$
\begin{aligned}
& \theta_{\mathrm{b}}=\frac{k^{2} \phi_{0}}{2} \phi_{0} \eta+D_{\mathrm{b}} k^{4} \eta^{3}, \\
& \theta_{\mathrm{c}}=\frac{k^{2} \phi_{0}}{2} \phi_{0} \eta+D_{\mathrm{c}} k^{4} \eta^{3},
\end{aligned}
$$

and

$$
\begin{gathered}
\delta_{\mathrm{b}}=-\frac{3}{4}\left(2 \phi_{0}+\Omega_{\mathrm{B}}\right)+A_{\mathrm{b}} k^{2} \eta^{2}, \\
\delta_{\mathrm{c}}=-\frac{3}{4}\left(2 \phi_{0}+\Omega_{\mathrm{B}}\right)+A_{\mathrm{c}} k^{2} \eta^{2} .
\end{gathered}
$$

The constants appearing in Eqs. (3.32), (3.33), (3.34), and (3.35) should satisfy

$$
\begin{gathered}
A_{\mathrm{b}, \mathrm{c}}=3 C_{\psi}-\frac{\phi_{0}}{4}, \\
4 D_{\mathrm{b}, \mathrm{c}}=C_{\phi},
\end{gathered}
$$

where the notation $A_{\mathrm{b}, \mathrm{c}}$ and $D_{\mathrm{b}, \mathrm{c}}$ means that the relations hold, separately, for the baryon and CDM integration constants. 
The anisotropic stress $\sigma_{\nu}$ appears both in Eqs. (2.37) and (2.38) and in Eq. (3.13), determining the difference between the two longitudinal fluctuations of the metric. Hence, from Eqs. (3.13) and (2.36) and (2.37) the neutrinos fluid variables are determined to be

$$
\begin{gathered}
\sigma_{\nu}=\frac{k^{2} \eta^{2}}{6 R_{\nu}}\left(\psi_{0}-\phi_{0}\right)-\frac{\Omega_{\mathrm{B}}}{4 R_{\nu}}+\frac{D_{\nu}}{15} k^{4} \eta^{4}, \\
\theta_{\nu}=k^{2}\left[\frac{\phi_{0}}{2}-\frac{\Omega_{\mathrm{B}}}{4} \frac{R_{\nu}-1}{R_{\nu}}\right]+D_{\nu} k^{4} \eta^{3}, \\
\delta_{\nu}=-\left(2 \phi_{0}+\Omega_{\mathrm{B}}\right)=A_{\nu} k^{2} \eta^{2},
\end{gathered}
$$

subject to the constraints

$$
\begin{gathered}
3 D_{\nu}-\frac{A_{\nu}}{4}=C_{\phi}-\frac{\phi_{0}}{15}+\frac{\Omega_{\mathrm{B}}}{30} \frac{R_{\nu}-1}{R_{\nu}}, \\
A_{\nu}=-\frac{\phi_{0}}{3}+\frac{\Omega_{\mathrm{B}}}{6} \frac{R_{\nu}-1}{R_{\nu}}+4 C_{\psi}, \\
C_{\phi}-C_{\psi}=-\frac{2}{5} D_{\nu} .
\end{gathered}
$$

The lowest order of Eq. (2.38) also implies

$$
\psi_{0}=\phi_{0}\left(1+\frac{2}{5} R_{\nu}\right)-\frac{\Omega_{\mathrm{B}}}{5}\left(R_{\nu}-1\right) .
$$

Up to now all the evolution equations for the fluid variables have been consistently solved in terms of a set of unknown constants satisfying a set of (algebraic) consistency conditions. From the remaining two Einstein Eqs. (3.4) and (3.9), two further constraints on the various constants can be obtained, namely

$$
C_{\phi}+2 C_{\psi}=2 D_{\nu} R_{\nu}+2\left(1-R_{\nu}\right) D_{\gamma}
$$

$$
\begin{aligned}
6 C_{\psi}+C_{\phi}= & -\frac{2}{15} R_{\nu} \phi_{0}+\frac{\Omega_{\mathrm{B}}}{15}\left(R_{\nu}-1\right)+\frac{1}{2}\left[A_{\nu} R_{\nu}\right. \\
& \left.+\left(1-R_{\nu}\right) A_{\gamma}\right] .
\end{aligned}
$$

Since two of the algebraic equations are not independent, the system of ten algebraic equations in ten unknowns becomes

$$
\begin{aligned}
& A_{\mathrm{b}}=A_{\mathrm{c}}=-\left[\frac{69-61 R_{\nu}-8 R_{\nu}^{2}}{60\left(25+2 R_{\nu}\right)} \Omega_{\mathrm{B}}+\frac{525+188 R_{\nu}+16 R_{\nu}^{2}}{60\left(25+2 R_{\nu}\right)} \phi_{0}\right], \\
& A_{\gamma}=-\left[-\frac{237+152 R_{\nu}+16 R_{\nu}^{2}}{90\left(25+2 R_{\nu}\right)} \Omega_{\mathrm{B}}+\frac{\left(525+188 R_{\nu}+16 R_{\nu}^{2}\right)}{45\left(25+2 R_{\nu}\right)} \phi_{0}\right], \\
& A_{\nu}=-\left[\frac{375-207 R_{\nu}-152 R_{\nu}^{2}-16 R_{\nu}^{3}}{90 R_{\nu}\left(25+2 R_{\nu}\right)} \Omega_{\mathrm{B}}+\frac{\left(525+188 R_{\nu}+16 R_{\nu}^{2}\right)}{45\left(25+2 R_{\nu}\right)} \phi_{0}\right], \\
& C_{\phi}=-\left[\frac{6-8 R_{\nu}+2 R_{\nu}^{2}}{45\left(25+2 R_{\nu}\right)} \Omega_{\mathrm{B}}+\frac{75+14 R_{\nu}-8 R_{\nu}^{2}}{90\left(25+2 R_{\nu}\right)} \phi_{0}\right], \quad C_{\psi}=-\left[\frac{69-61 R_{\nu}-8 R_{\nu}^{2}}{180\left(25+2 R_{\nu}\right)} \Omega_{\mathrm{B}}+\frac{\left(75+79 R_{\nu}+8 R_{\nu}^{2}\right)}{90\left(25+2 R_{\nu}\right)} \phi_{0}\right], \\
& D_{\gamma}=-\left[\frac{\left(25+8 R_{\nu}\right)}{20\left(25+2 R_{\nu}\right)} \phi_{0}-\frac{\left(7+8 R_{\nu}\right)}{40\left(25+2 R_{\nu}\right)} \Omega_{\mathrm{B}}\right], \quad D_{\nu}=-\left[\frac{45-29 R_{\nu}-16 R_{\nu}^{2}}{72 R_{\nu}\left(25+2 R_{\nu}\right)} \Omega_{\mathrm{B}}+\frac{\left(65+16 R_{\nu}\right)}{36\left(25+2 R_{\nu}\right)} \phi_{0}\right], \\
& D_{\mathrm{b}}=D_{\mathrm{c}}=\frac{\phi_{0}}{2} k^{2} \eta-\left[\frac{6-8 R_{\nu}+2 R_{\nu}^{2}}{180\left(25+2 R_{\nu}\right)} \Omega_{\mathrm{B}}+\frac{75+14 R_{\nu}-8 R_{\nu}^{2}}{360\left(25+2 R_{\nu}\right)} \phi_{0}\right] .
\end{aligned}
$$

Recalling the form of the standard solution reported in Eqs. (3.16), (3.17), (3.18), (3.19), (3.20), (3.21), (3.22), (3.23), and (3.24), and using the results obtained so far, the full solution can be written as

$$
\begin{aligned}
& \delta_{\mathrm{b}, \mathrm{c}}=\bar{\delta}_{\mathrm{b}, \mathrm{c}}-\frac{3}{4} \Omega_{\mathrm{B}}-\left[\frac{69-61 R-8 R_{\nu}^{2}}{60\left(25+2 R_{\nu}\right)}\right] \Omega_{\mathrm{B}} k^{2} \eta^{2}, \\
& \delta_{\gamma}=\bar{\delta}_{\gamma}-\Omega_{\mathrm{B}}+\left[\frac{237+152 R_{\nu}+16 R_{\nu}^{2}}{90\left(25+2 R_{\nu}\right)}\right] \Omega_{\mathrm{B}} k^{2} \eta^{2},
\end{aligned}
$$

$$
\begin{aligned}
\delta_{\nu}= & \bar{\delta}_{\nu}-\Omega_{\mathrm{B}} \\
& -\left[\frac{375-207 R_{\nu}-152 R_{\nu}^{2}-16 R_{\nu}^{3}}{90 R_{\nu}\left(25+2 R_{\nu}\right)}\right] \Omega_{\mathrm{B}} k^{2} \eta^{2},
\end{aligned}
$$

$$
\theta_{\gamma}=\bar{\theta}_{\gamma}-\left[\frac{\Omega_{\mathrm{B}}}{4} k^{2} \eta+\frac{\left(7+8 R_{\nu}\right)}{40(25+2 R)} \Omega_{\mathrm{B}}\right] k^{4} \eta^{3},
$$




$$
\begin{gathered}
\theta_{\nu}=\bar{\theta}_{\nu}-\frac{\Omega_{\mathrm{B}}}{4} \frac{R_{\gamma}}{R_{\nu}} k^{2} \eta-\left[\frac{45-29 R_{\nu}-16 R_{\nu}^{2}}{72 R_{\nu}\left(25+2 R_{\nu}\right)} \Omega_{\mathrm{B}}\right] k^{4} \eta^{3}, \\
\theta_{\mathrm{b}}=\theta_{\mathrm{c}}=\bar{\theta}_{\mathrm{b}}-\left[\frac{6-8 R_{\nu}+2 R_{\nu}^{2}}{180\left(25+2 R_{\nu}\right)} \Omega_{\mathrm{B}}\right] k^{4} \eta^{3}
\end{gathered}
$$

where the overline in the various quantities in the lefthand side denote the adiabatic solution derived above.

Concerning these results, the following comments are in order:

(i) unlike the standard case, the difference between the two constant modes of the longitudinal fluctuations of the metric is also determined by the magnetic energy density; in the limit $\Omega_{\mathrm{B}} \rightarrow 0$, the standard expression (obtained in the previous subsection) is recovered;

(ii) the adiabaticity condition is enforced to lowest order in $k \eta$, but it is violated at the next-toleading order;

(iii) the force-free approximation neglects, by assumption, the Lorentz force and this is the rationale for the equality, for instance, between $\theta_{\mathrm{b}}$ and $\theta_{\mathrm{c}}$ : in the absence of Lorentz force the magnetic field does not impart gradients to the baryonic component of the fluid;

(iv) the presence of magnetic fields introduces a further source of anisotropic stress as it can be appreciated from the chain of (first-order) relations $\psi-\phi \simeq \bar{\psi}-\bar{\phi}-\Omega_{\mathrm{B}}\left(R_{\nu}-1\right) / 5$ which follows from Eq. (3.44).

Since the adiabaticity condition is only realized to lowest order in $k \eta$ it is legitimate to name the solution presented in Eqs. (3.48), (3.49), (3.50), (3.51), (3.52), (3.53), (3.54), and (3.55) quasiadiabatic. The corrections to adiabaticity are of order $\Omega_{\mathrm{B}} k^{2} \eta^{2}$, so they are suppressed outside the horizon and also, by virtue of Eq. (3.7), by $\Omega_{\mathrm{B}}$. Notice that three regimes emerge naturally: the quasiadiabatic regime where $\Omega_{\mathrm{B}}(k) \leq \phi_{0}(k)$, the isocurvature regime $\Omega_{\mathrm{B}}(k)>\phi_{0}(k)$ and the fully adiabatic regime, i.e., $\Omega_{\mathrm{B}}(k) \ll \phi_{0}(k)$.

The force-free limit may be (approximately) realized in nature in some specific (but rather interesting) cases. For instance, it could happen that magnetic fields are generated in a so-called maximally helical configuration. Recall, in fact, that given a magnetic field configuration, in a MHD description, it is always possible to define the related magnetic helicity [25], which is

$$
\int d^{3} x \vec{A} \cdot \vec{B}
$$

Now, at finite conductivity, the evolution of the magnetic helicity can be written as

$$
\frac{d}{d \eta} \int d^{3} x \vec{A} \cdot \vec{B}=-\frac{1}{4 \pi \sigma} \int d^{3} x \vec{B} \cdot \vec{\nabla} \times \vec{B},
$$

where the expression appearing in the right-hand side is called magnetic gyrotropy [25] and measures the number of contact points in the magnetic field lines. Clearly, if the magnetic gyrotropy is maximal, the orthogonal combination, namely, the Lorentz force $\vec{\nabla} \times \vec{B} \times \vec{B}$, will be minimal or even zero. Maximally helical configurations can be very relevant in the development of MHD turbulence [25] and have been extensively studied in a cosmological context (see also [20] for a general discussion and [37] for a recent paper). In particular it should be noticed that helical configurations can be produced at the electroweak time by coupling the hypercharge field to a pseudoscalar [38] (see also [39]).

\section{Contribution of the Lorentz force}

Consider now the case when the magnetic field is not force-free. The major difference with the force-free case is that the evolution equation for the peculiar velocity of the baryons receives a contribution from the magnetic field gradient. Because of the tightly coupled dynamics of baryons and photons, also the photon peculiar velocity will be modified. Since photons are related to neutrinos by the Einstein equations, the whole solution will be modified.

Equation (2.26) can be written, after multiplying both sides by the scale factor $a(\eta)$, as

$$
\left(a \theta_{\mathrm{b}}\right)^{\prime}=k^{2} \phi_{0} a+\frac{F_{\mathrm{B}}}{4 \pi \rho_{\mathrm{b}} a^{3}},
$$

where it has been assumed that $\phi$ has, to lowest order, a constant solution denoted, as usual, by $\phi_{0}$. The second term in the right-hand side of Eq. (3.58) is then constant and direct integration gives

$$
\theta_{\mathrm{b}}=\frac{\phi_{0}}{2} k^{2} \eta+\frac{F_{\mathrm{B}}}{4 \pi \rho_{\mathrm{b}} a^{3}} .
$$

From Eq. (2.27), using Eq. (3.59), the density contrast can be determined up to a constant, which is fixed from the adiabaticity condition:

$$
\delta_{\mathrm{b}}=-\frac{3}{2} \phi_{0}-\frac{3}{4} \Omega_{\mathrm{B}}-\frac{F_{\mathrm{B}}}{4 \pi \rho_{\mathrm{b}} a^{3}} \eta .
$$

The Hamiltonian constraint of Eq. (3.1) and the equation for the peculiar velocity of the photons imply

$$
\begin{gathered}
\delta_{\nu} \simeq \delta_{\gamma} \simeq-2 \phi_{0}-\Omega_{\mathrm{B}}, \\
\theta_{\gamma}=\left(\frac{\phi_{0}}{2}-\frac{\Omega_{\mathrm{B}}}{4}\right) k^{2} \eta .
\end{gathered}
$$


The CDM Eqs. (2.34) and (2.35) will then turn out to be

$$
\delta_{\mathrm{c}} \simeq-2 \phi_{0}, \quad \theta_{\mathrm{c}}=\frac{\phi_{0}}{2} k^{2} \eta .
$$

The anisotropic stress of the neutrinos can be determined by solving Eq. (3.13):

$$
\sigma_{\nu}=\frac{k^{2} \eta^{2}}{6 R}\left(\psi_{0}-\phi_{0}\right)-\frac{3}{4} \frac{\sigma_{\mathrm{B}}}{R_{\nu}} .
$$

Inserting Eq. (3.64) into Eq. (2.37) the peculiar velocity of the neutrino fluid can be determined to be

$$
\theta_{\nu}=\left(\frac{\phi_{0}}{2}-\frac{\Omega_{\mathrm{B}}}{4}+\frac{3}{4} \frac{\sigma_{\mathrm{B}}}{R_{\nu}}\right) k^{2} \eta .
$$

Finally from Eq. (2.38), using Eq. (3.64), the difference between the two longitudinal fluctuations of the metric can be derived:

$$
\psi_{0}=\phi_{0}\left(1+\frac{2}{5} R_{\nu}\right)-\frac{R_{\nu}}{5} \Omega_{\mathrm{B}}+\frac{3}{5} \sigma_{\mathrm{B}} .
$$

The momentum constraint derived in Eq. (3.4) is satisfied by virtue of the following identity:

$$
\sigma_{\mathrm{B}} k^{2} \eta-\frac{\Omega_{\mathrm{B}}}{3}+\Omega_{\mathrm{b}} \frac{F_{\mathrm{B}}}{4 \pi \rho_{\mathrm{b}} a^{3}}=0 .
$$

Notice also that the contribution of the Lorentz force is suppressed, for $k \rightarrow 0$ with respect to $\Omega_{\mathrm{B}}$ since, roughly, $F_{\mathrm{B}} \sim k^{2} \Omega_{\mathrm{B}}$.

\section{SYNCHRONOUS GAUGE DESCRIPTION}

In the longitudinal description, the gauge freedom is completely specified and, hence, the so-called gauge modes are absent. ${ }^{4}$ As a consequence [40], in the longitudinal gauge the metric fluctuations correspond directly to a particular set of gauge-invariant combinations, namely, the Bardeen potentials [3]. Having said this, it is important to appreciate that longitudinal and synchronous descriptions should be regarded as complementary and not as opposite. The synchronous description, for instance, is more convenient when modes based on anisotropic stresses are discussed, as in the case of the present analysis. Furthermore, the known (old) problem of spurious gauge modes is completely settled by now since the work of Press and Vishniac [6]. In some specific calculations it turns out to be useful to profit from the extra gauge freedom inherent in the synchronous description. There are examples in the literature of isocurvature modes that are divergent, at early times, in the longitu-

\footnotetext{
${ }^{4}$ According to the usual nomenclature, gauge modes are those modes arising when gauge freedom is not completely fixed, as in the synchronous gauge.
}

dinal gauge, but which are perfectly physical and regular in the synchronous coordinate system [36]. Finally, it is important to consider the formulation of the problem of initial conditions in the synchronous gauge, since various numerical codes solving the Boltzmann hierarchy use, indeed, the synchronous description.

In the synchronous description the line element can be consistently perturbed in the form:

$$
d s^{2}=a^{2}(\eta) d \eta^{2}-a^{2}(\eta)\left(\delta_{i j}-h_{i j}\right) d x^{i} d x^{j},
$$

where the perturbed element of the metric is given by

$$
\delta g_{i j}=a^{2} h_{i j}, \quad \delta g^{i j}=-\frac{h^{i j}}{a^{2}} .
$$

Sometimes the parametrization $\delta g_{i j}=2 a^{2}\left(\psi_{\mathrm{s}} \delta_{i j}+\right.$ $\left.\partial_{i} \partial_{j} E_{\mathrm{s}}\right)$ is also employed [40]. The connection between the two parametrizations is immediate by separating, in Fourier space, the trace of the perturbation from its traceless part:

$h_{i j}(\eta, \vec{x})=\int d^{3} k e^{i \vec{k} \cdot \vec{x}}\left[\hat{k}_{i} \hat{k}_{j} h(k, \eta)+6 \xi(k, \eta)\left(\hat{k}_{i} \hat{k}_{j}-\frac{1}{3} \delta_{i j}\right)\right]$,

where $\hat{k}^{i}=k^{i} /|\vec{k}|$. Clearly, by performing an infinitesimal gauge transformation the $\delta g_{00}$ and $\delta g_{0 i}$ parts of the metric (which are not perturbed in the synchronous parametrization) also transform. The gauge modes can be exactly identified by requiring that $\delta g_{00}=0$ and $\delta g_{i j}=0$, for gauge transformations that preserve the synchronous nature of the coordinate system. The first gauge mode corresponds to a spatial reparametrization of the constant-time hypersurfaces. As a consequence, in this mode the metric perturbation is constant and the matter density unperturbed. The second gauge mode corresponds to a spatially dependent shift in the time direction.

With the notation of Eqs. (4.2) and (4.3), the perturbed Einstein equations become

$$
\begin{aligned}
k^{2} \xi-\frac{\mathcal{H}}{2} h^{\prime}= & \frac{3}{2} \mathcal{H}^{2}\left[R_{\nu} \delta_{\nu}+\left(1-R_{\nu}\right) \delta_{\gamma}+\Omega_{\mathrm{B}}\right. \\
& \left.+\Omega_{\mathrm{b}} \delta_{\mathrm{b}}+\Omega_{\mathrm{c}} \delta_{\mathrm{c}}\right], \\
k^{2} \xi^{\prime}= & -\frac{3}{2} \mathcal{H}^{2}\left\{\frac{F_{\mathrm{B}}}{4 \pi \sigma \rho}+\frac{4}{3}\left[R_{\nu} \theta_{\nu}+\left(1-R_{\nu}\right) \theta_{\gamma}\right]\right. \\
& \left.+\Omega_{\mathrm{b}} \theta_{\mathrm{b}}+\Omega_{\mathrm{c}} \theta_{\mathrm{c}}\right\}
\end{aligned}
$$

$$
h^{\prime \prime}+2 \mathcal{H} h^{\prime}-2 k^{2} \xi=9 \mathcal{H}^{2}\left\{\frac{1}{3}\left[R_{\nu} \delta_{\nu}+\left(1-R_{\nu}\right) \delta_{\gamma}\right]+\frac{\Omega_{\mathrm{B}}}{3}\right\},
$$




$$
\begin{aligned}
h^{\prime \prime} & +6 \xi^{\prime \prime}+2 \mathcal{H} h^{\prime}+12 \mathcal{H} \xi^{\prime}-2 k^{2} \xi \\
& =9 \mathcal{H}^{2}\left[\sigma_{\mathrm{B}}+\frac{4}{3} R_{\nu} \sigma_{\nu}\right],
\end{aligned}
$$

where the background Eqs. (2.3), (2.4), and (2.5) have already been used to eliminate the energy and pressure densities. ${ }^{5}$ By combining appropriately Eqs. (4.4) and (4.6), it is possible to obtain a further useful equation

$$
\begin{aligned}
h^{\prime \prime}+\mathcal{H} h^{\prime}= & 3 \mathcal{H}^{2}\left[2 R_{\nu} \delta_{\nu}+2\left(1-R_{\nu}\right) \delta_{\gamma}+2 \Omega_{\mathrm{B}}\right. \\
& \left.+\Omega_{\mathrm{b}} \delta_{\mathrm{b}}+\Omega_{\mathrm{c}} \delta_{\mathrm{c}}\right] .
\end{aligned}
$$

The evolution equations of the peculiar velocities and density contrasts of the various species of the plasma can be obtained by perturbing the covariant conservation of the energy-momentum tensor. The result is the following:

$$
\begin{gathered}
\delta_{\nu}^{\prime}=-\frac{4}{3} \theta_{\nu}+\frac{2}{3} h^{\prime}, \\
\delta_{\gamma}^{\prime}=-\frac{4}{3} \theta_{\gamma}+\frac{2}{3} h^{\prime}, \\
\delta_{\mathrm{b}}^{\prime}=-\theta_{\mathrm{b}}+\frac{1}{2} h^{\prime}, \\
\delta_{\mathrm{c}}^{\prime}=-\theta_{\mathrm{c}}+\frac{h^{\prime}}{2}, \\
\theta_{\nu}^{\prime}=-k^{2} \sigma_{\nu}+\frac{k^{2}}{4} \delta_{\nu}, \\
\theta_{\gamma}^{\prime}=\frac{k^{2}}{4} \delta_{\gamma}, \\
\theta_{\mathrm{b}}^{\prime}=-\mathcal{H} \theta_{\mathrm{b}}+\frac{F_{\mathrm{B}}}{4 \pi \rho_{\mathrm{b}} a^{4}}, \\
\theta_{\mathrm{c}}^{\prime}=-\mathcal{H} \theta_{\mathrm{c}}, \\
\sigma_{\nu}^{\prime}=\frac{4}{15} \theta_{\nu}-\frac{3}{10} k \mathcal{F}{ }_{\nu 3}+\frac{2}{15} h^{\prime}+\frac{4}{5} \xi^{\prime} .
\end{gathered}
$$

As anticipated the synchronous gauge modes can be made harmless by eliminating the constant solution for $h$ and by fixing, for instance, the CDM velocity field to zero. These two requirements specify the coordinate system completely.

\footnotetext{
${ }^{5}$ In this section the density contrasts and the peculiar velocity field are named in the same way as in the longitudinal gauge. It is understood that the density contrasts and the peculiar velocity fields are not equal in the two gauges and are related by the transformations listed below [see Eqs. (4.26) and (4.28)].
}

The force-free solution can also be obtained within the synchronous description. In particular, for the metric fluctuations the solution is the following:

$$
\begin{gathered}
\xi=-2 C+\frac{R_{\nu}-1}{5} \Omega_{\mathrm{B}}+\left[\frac{5+4 R_{\nu}}{6\left(15+4 R_{\nu}\right)} C\right. \\
\left.-\frac{R_{\nu}-1}{60} \Omega_{\mathrm{B}}\right] k^{2} \eta^{2}, \\
h=\left[\frac{\Omega_{\mathrm{B}}}{10}-C\right] k^{2} \eta^{2} .
\end{gathered}
$$

The constant introduced in Eqs. (4.18) and (4.19) has been defined in order to match the standard notation usually employed in the literature (see for instance [5]) to characterize the adiabatic (inflationary) mode. By solving Eqs. (4.9), (4.10), (4.11), (4.12), (4.13), (4.14), (4.15), (4.16), and (4.17) the solution for the density contrasts

$$
\begin{gathered}
\delta_{\gamma}=-\Omega_{\mathrm{B}}+\left[\frac{2 R_{\nu}+3}{30} \Omega_{\mathrm{B}}-\frac{2}{3} C\right] k^{2} \eta^{2}, \\
\delta_{\nu}=-\Omega_{\mathrm{B}}+\left[\frac{2 R_{\nu}^{2}+3 R_{\nu}-5}{30 R_{\nu}} \Omega_{\mathrm{B}}-\frac{2}{3} C\right] k^{2} \eta^{2}, \\
\delta_{\mathrm{b}}=-\frac{3}{4} \Omega_{\mathrm{B}}+\left[\frac{R_{\nu}-1}{20} \Omega_{\mathrm{B}}-\frac{C}{2}\right] k^{2} \eta^{2}, \\
\delta_{\mathrm{c}}=-\frac{3}{4} \Omega_{\mathrm{B}}+\left[\frac{R_{\nu}-1}{20} \Omega_{\mathrm{B}}-\frac{C}{2}\right] k^{2} \eta^{2},
\end{gathered}
$$

and for the peculiar velocities

$$
\begin{gathered}
\theta_{\gamma}=-\frac{\Omega_{\mathrm{B}}}{4} k^{2} \eta+\left[\frac{2 R_{\nu}+3}{360} \Omega_{\mathrm{B}}-\frac{C}{18}\right], \\
\theta_{\nu}=-\frac{\Omega_{\mathrm{B}}}{4} k^{2} \eta+\left[\frac{2 R_{\nu}^{2}+7 R_{\nu}-9}{360 R_{\nu}} \Omega_{\mathrm{B}}-\frac{23+4 R_{\nu}}{15+4 R_{\nu}}\right],
\end{gathered}
$$

can be obtained. The solution derived in the previous equations of the present section can be transformed into the longitudinal gauge by using the appropriate transformation, i.e.,

$$
\begin{aligned}
\phi_{\mathrm{L}} & =-\frac{1}{2 k^{2}}\left[(6 \xi+h)^{\prime \prime}+\mathcal{H}(6 \xi+h)^{\prime}\right], \\
\psi_{\mathrm{L}} & =-\xi+\frac{\mathcal{H}}{2 k^{2}}\left(6 \xi^{\prime}+h^{\prime}\right), \\
\delta_{\mathrm{L}}^{(\lambda)} & =\delta_{\mathrm{s}}^{(\lambda)}+3\left(w_{\lambda}+1\right) \frac{\mathcal{H}}{2 k^{2}}\left(h^{\prime}+6 \xi^{\prime}\right), \\
\theta_{\mathrm{L}}^{(\lambda)} & =\theta_{\mathrm{s}}^{(\lambda)}-\frac{1}{2}\left(h^{\prime}+6 \xi^{\prime}\right),
\end{aligned}
$$

where the subscripts refer to the quantities evaluated either in the longitudinal or in the synchronous gauge. By setting $\Omega_{\mathrm{B}}=0$ in Eqs. (4.18), (4.19), (4.20), (4.21), 
(4.22), (4.23), (4.24), and (4.25), we recover the standard adiabatic mode discussed in the appendix with the constant $C$ determined as

$$
C=\frac{15+4 R_{\nu}}{20} \phi_{0},
$$

where $\phi_{0}$ is the value of the adiabatic mode in the longitudinal gauge discussed previously. Of course, the solutions of the longitudinal gauge can also be directly transformed into the synchronous gauge using the appropriate transformation, which can be easily derived:

$$
\begin{aligned}
\xi= & -\psi_{L}-\frac{\mathcal{H}}{a} \int a \phi_{L} d \eta, \\
h= & 6 \psi_{L}+6 \frac{\mathcal{H}}{a} \int a \phi_{L} d \eta, \\
& -2 k^{2} \int \frac{d \eta^{\prime \prime}}{a\left(\eta^{\prime \prime}\right)} \int^{\eta^{\prime \prime}} a\left(\eta^{\prime}\right) \phi_{L}\left(\eta^{\prime}\right) d \eta^{\prime}, \\
\delta_{s}^{(\lambda)}= & \delta_{L}^{(\lambda)}+3 \mathcal{H}(w+1) \frac{1}{a} \int \phi_{L} a d \eta, \\
\theta_{s}^{(\lambda)}= & \theta_{L}^{(\lambda)}-\frac{k^{2}}{a} \int a \phi_{L} d \eta .
\end{aligned}
$$

Up to now we always studied adiabatic (or quasiadiabatic) solutions. However, also isocurvature solutions can be generalized to include fully inhomogeneous magnetic fields. In order to look for isocurvature solutions it is useful to recall that in looking for perturbative solutions (both in the longitudinal and in the synchronous gauge) there are various small parameters. One is certainly $k \eta$, which is small outside the horizon. However, also $\Omega_{\mathrm{b}}$ and $\Omega_{\mathrm{c}}$ are small parameters deep within the radiationdominated epoch. Let us make this statement more precise by considering the scale factor

$$
a(\eta)=\left[\left(\frac{\eta}{\eta_{1}}\right)+\left(\frac{\eta}{\eta_{1}}\right)^{2}\right],
$$

interpolating between the radiation-dominated phase for $\eta \ll \eta_{1}$ and the matter-dominated epoch for $\eta \gg \eta_{1}$. In this case we can also write

$$
\begin{aligned}
\Omega_{\mathrm{b}, \mathrm{c}} & =\bar{\Omega}_{\mathrm{b}, \mathrm{c}} \frac{a(\eta)}{a(\eta)+1}, \\
\Omega_{\mathrm{B}} & =\bar{\Omega}_{\mathrm{B}} \frac{1}{a(\eta)+1},
\end{aligned}
$$

where the subscripts in Eq. (4.30) refer either to baryons or to CDM and where we took, for simplicity, $\eta_{1}=1$.

We can then insert Eqs. (4.29), (4.30), and (4.31) into the evolution equations for the perturbations in the synchronous gauge, regarding $\Omega_{\mathrm{b}, \mathrm{c}}$ as small parameters deep within the radiation-dominated epoch $(\eta \rightarrow 0)$. The result of this procedure is summarized by the following solutions, which are valid in the case $\sigma_{\mathrm{B}}=\Omega_{\mathrm{B}} / 3$ :

$$
\begin{gathered}
h \simeq\left(-4 \bar{\Omega}_{\mathrm{b}} \eta+6 \bar{\Omega}_{\mathrm{b}} \eta^{2}\right), \\
\xi \simeq \frac{2}{3} \bar{\Omega}_{\mathrm{b}}-\bar{\Omega}_{\mathrm{b}} \eta^{2},
\end{gathered}
$$

for the metric perturbations and

$$
\begin{gathered}
\delta_{\gamma} \simeq\left(-\frac{8}{3} \bar{\Omega}_{\mathrm{b}} \eta+4 \bar{\Omega}_{\mathrm{b}} \eta^{2}\right),-\bar{\Omega}_{\mathrm{B}}\left(1-\eta+\eta^{3}\right), \\
\delta_{\mathrm{b}}=\left(1-2 \bar{\Omega}_{\mathrm{b}}+3 \bar{\Omega}_{\mathrm{b}} \eta^{2}\right), \\
\delta_{\nu} \simeq\left(-\frac{8}{3} \bar{\Omega}_{\mathrm{b}} \eta+4 \bar{\Omega}_{\mathrm{b}} \eta^{2}\right) \\
\delta_{\mathrm{c}} \simeq 2 \bar{\Omega}_{\mathrm{b}}+3 \bar{\Omega}_{\mathrm{b}} \eta^{2}, \\
\theta_{\gamma} \simeq-\frac{1}{3} \bar{\Omega}_{\mathrm{b}} k^{2} \eta^{2}-\frac{k^{2}}{16} \bar{\Omega}_{\mathrm{B}}\left(4 \eta-2 \eta^{2}+\eta^{4}\right), \\
\theta_{\nu} \simeq-\frac{1}{3} \bar{\Omega}_{\mathrm{b}} k^{2} \eta^{2}-\frac{k^{2}}{16} \bar{\Omega}_{\mathrm{B}}\left(4 \eta-2 \eta^{2}+\eta^{4}\right) \frac{R_{\gamma}}{R_{\nu}}, \\
\theta_{\mathrm{c}}=0, \\
\frac{2}{3} \frac{\bar{\Omega}_{\mathrm{b}}}{2 R_{\nu}+15} k^{2} \eta^{3}-\frac{\bar{\Omega}_{\mathrm{B}}}{4 R_{\nu}}\left(1-\eta+\eta^{3}\right),
\end{gathered}
$$

for the fluid quantities. Clearly, for this mode, the adiabaticity condition is not satisfied. Furthermore, by transforming the solution to the Newtonian gauge, it is easy to check that the longitudinal fluctuations of the metric vanish for $\eta \rightarrow 0$. In the limit $\Omega_{\mathrm{B}} \rightarrow 0$ this mode reduces to the baryon isocurvature mode already discussed in Ref. [36]. A similar solution can be obtained by changing $\bar{\Omega}_{\mathrm{b}} \rightarrow \bar{\Omega}_{\mathrm{c}}$.

\section{TIGHT-COUPLING EXPANSION IN MAGNETOACTIVE PLASMAS}

One of the analytical tools often employed in the theory of the CMB anisotropies is the so-called tightcoupling expansion [41]. Defining the differential optical depth as done in Eq. (2.28) the exact tight-coupling limit is realized when $\sigma_{\mathrm{T}} \rightarrow \infty$ and $1 / \tau^{\prime} \rightarrow 0$. If tight coupling is exact, photons and baryons are synchronized so well that the photon phase-space distribution is isotropic in the baryon rest frame. Since the photon distribution is isotropic, the resulting radiation is not polarized. However, as the decoupling time approaches there is a regime where $\left|1 / \tau^{\prime}\right|<1$ without being zero. The idea is then to tailor a systematic expansion in powers of $\left|1 / \tau^{\prime}\right|$ and to consider not only the zeroth order but also higher orders depending upon the accuracy required by the problem. The evolution equations to be discussed are essentially the radiative transfer equations which are, in turn, the Boltzmann 
equations written in terms of the brightness perturbations. Within the notation employed in the present paper, these equations are derived in Eqs. (A26)-(A28) of the appendix. The brightness perturbations should be studied for each of the four Stokes parameters. The brightness perturbations $\Delta_{\mathrm{I}}$, connected with the first Stokes parameter I, are the temperature fluctuations, i.e., fluctuations in the intensity of the radiation field. As discussed in the appendix, recalling that $\hat{n}$ is the direction of the comoving-three-momentum of the photon and defining $\mu=\hat{k} \cdot \hat{n}$, the evolution of the brightness perturbation is$$
\Delta_{\mathrm{I}}^{\prime}+i k \mu \Delta_{\mathrm{I}}=\left(\psi^{\prime}-i k \mu \phi\right)+\tau^{\prime}\left[-\Delta_{\mathrm{I}}+\Delta_{\mathrm{I} 0}+\mu v_{\mathrm{b}}\right.
$$

$$
\left.-\frac{1}{2} P_{2}(\mu) S_{0}\right] \text {, }
$$

where, in our notation, $v_{\mathrm{b}}=\theta_{\mathrm{b}} /(i k)$ and

$$
S_{0}=\Delta_{\mathrm{I} 2}+\Delta_{\mathrm{Q} 0}+\Delta_{\mathrm{Q} 2},
$$

where, with obvious notation (discussed in detail in the appendix)

$$
\begin{aligned}
\Delta_{\mathrm{I}}(\vec{k}, \hat{n}, \eta) & =\sum_{\ell}(-i)^{\ell}(2 \ell+1) \Delta_{\mathrm{I} \ell}(\vec{k}, \eta) P_{\ell}(\mu), \\
\Delta_{\mathrm{Q}}(\vec{k}, \hat{n}, \eta) & =\sum_{\ell}(-i)^{\ell}(2 \ell+1) \Delta_{\mathrm{Q} \ell}(\vec{k}, \eta) P_{\ell}(\mu),
\end{aligned}
$$

$\Delta_{\mathrm{I} \ell}$ and $\Delta_{\mathrm{Q} \ell}$ being the $\ell$ th multipole of the brightness function $\Delta_{\mathrm{I}}$ and $\Delta_{\mathrm{Q}}$.

The function $P_{2}(\mu)=\left(3 \mu^{2}-1\right) / 2$ in Eq. (5.2) is the Legendre polynomial of second order, which appears in the collision operator of the Boltzmann equation for the photons due to the directional nature of Thompson scattering. The evolution equations for the brightness perturbations connected with the Q and U Stokes parameters are

$$
\begin{gathered}
\Delta_{\mathrm{Q}}^{\prime}+i k \mu \Delta_{\mathrm{Q}}=\tau^{\prime}\left\{-\Delta_{\mathrm{Q}}+\frac{1}{2}\left[1-P_{2}(\mu)\right] S_{0}\right\}, \\
\Delta_{\mathrm{U}}^{\prime}+i k \mu \Delta_{\mathrm{U}}=-\tau^{\prime} \Delta_{\mathrm{U}} .
\end{gathered}
$$

Finally, in order to close the system, the baryon evolution equation should be added using the definition $v_{\mathrm{b}}=$ $\theta_{\mathrm{b}} /(i k)$ either in Eq. (2.26) or in (3.8):

$$
v_{\mathrm{b}}^{\prime}+\mathcal{H} v_{\mathrm{b}}=-i k \phi-\frac{\tau^{\prime}}{\alpha}\left[3 i \Delta_{\mathrm{I} 1}+v_{\mathrm{b}}\right]-i W_{B}(k),
$$

where

$$
\begin{gathered}
\mathcal{W}_{B}(k)=\frac{F_{\mathrm{B}}}{4 \pi k \rho_{\mathrm{b}} a^{4}}, \\
\alpha=\frac{3}{4} \frac{\rho_{\mathrm{b}}}{\rho_{\gamma}} .
\end{gathered}
$$

In (5.6) the dipole of the brightness function appears directly. In fact, using the results derived in the appendix, the following chain of identities holds:

$$
\theta_{\gamma}=\frac{3}{4} k \mathcal{F}_{\gamma 1}=3 \Delta_{\mathrm{I} 1},
$$

where $\mathcal{F}_{\gamma 1}$ is the dipole of the photon phase-space distribution and $\Delta_{\mathrm{I} 1}$ is the monopole of the brightness perturbation.

The idea is now to expand Eqs. (5.1) and (5.4) in powers of the small parameter $\epsilon=\left|1 / \tau^{\prime}\right|$, i.e., the inverse of the differential optical depth of Thompson scattering. Before doing the expansion, it is useful to derive the hierarchy for the brightness functions in full analogy with what is discussed in the appendix for the case of the neutrino phase-space distribution. To this aim, each side of Eqs. (5.1), (5.2), (5.3), (5.4), and (5.6) will be multiplied by the various Legendre polynomials and the integration over $\mu$ will be performed. Noticing that, from the orthonormality relation for Legendre polynomials [Eq. (A10) of the appendix],

$$
\int_{-1}^{1} P_{\ell}(\mu) \Delta_{\mathrm{I}} d \mu=2(-i)^{\ell} \Delta_{\mathrm{I} \ell}
$$

and recalling that

$$
\begin{array}{ll}
P_{0}(\mu)=1, & P_{1}(\mu)=\mu, \quad P_{2}(\mu)=\frac{1}{2}\left(3 \mu^{2}-1\right), \\
& P_{3}(\mu)=\frac{1}{2}\left(5 \mu^{3}-3 \mu\right),
\end{array}
$$

Eqs. (5.1), (5.2), (5.3), (5.4), (5.5), and (5.6) allow the determination of the first three sets of equations for the hierarchy of the brightness. More specifically, multiplying Eqs. (5.1), (5.2), (5.3), (5.4), and (5.6) by $P_{0}(\mu)$ and integrating over $\mu$, the following relations can be obtained:

$$
\begin{gathered}
\Delta_{\mathrm{I} 0}^{\prime}+k \Delta_{\mathrm{I} 1}=\psi^{\prime}, \\
\Delta_{\mathrm{Q} 0}^{\prime}+k \Delta_{\mathrm{Q} 1}=\frac{\tau^{\prime}}{2}\left[\Delta_{\mathrm{Q} 2}+\Delta_{\mathrm{I} 2}-\Delta_{\mathrm{Q} 0}\right] \\
\boldsymbol{v}_{b}^{\prime}+\mathcal{H} v_{\mathrm{b}}=-i k \phi-\frac{\tau^{\prime}}{\alpha}\left(3 i \Delta_{\mathrm{I} 1}+v_{b}\right)-\frac{i}{a} W_{B}(k) .
\end{gathered}
$$

If Eqs. (5.1), (5.2), (5.3), (5.4), and (5.6) are multiplied by $P_{1}(\mu)$, the integration over $\mu$ of the various terms implies

$$
-\Delta_{\mathrm{I} 1}^{\prime}-\frac{2}{3} k \Delta_{\mathrm{I} 2}+\frac{k}{3} \Delta_{\mathrm{I} 0}=-\frac{k}{3} \phi+\tau^{\prime}\left[\Delta_{\mathrm{I} 1}+\frac{1}{3 i} v_{\mathrm{b}}\right],
$$

$$
\begin{gathered}
-\Delta_{\mathrm{Q} 1}^{\prime}-\frac{2}{3} k \Delta_{\mathrm{Q} 2}+\frac{k}{3} \Delta_{\mathrm{Q} 0}=\tau^{\prime} \Delta_{\mathrm{Q} 1}, \\
\boldsymbol{v}_{b}^{\prime}+\mathcal{H} \boldsymbol{v}_{b}=-i k \phi-\frac{\tau^{\prime}}{\alpha}\left(3 i \Delta_{\mathrm{I} 1}+v_{b}\right)-\frac{i}{a} W_{B}(k) .
\end{gathered}
$$

The same procedure, using $P_{2}(\mu)$, leads to 


$$
\begin{gathered}
-\Delta_{\mathrm{I} 2}^{\prime}-\frac{3}{5} k \Delta_{\mathrm{I} 3}+\frac{2}{5} k \Delta_{\mathrm{I} 1}=\tau^{\prime}\left[\frac{9}{10} \Delta_{\mathrm{I} 2}-\frac{1}{10}\left(\Delta_{\mathrm{Q} 0}+\Delta_{\mathrm{Q} 2}\right)\right], \\
-\Delta_{\mathrm{Q} 2}^{\prime}-\frac{3}{5} k \Delta_{\mathrm{Q} 3}+\frac{2}{5} k \Delta_{\mathrm{Q} 1}=\tau^{\prime}\left[\frac{9}{10} \Delta_{\mathrm{Q} 2}-\frac{1}{10}\left(\Delta_{\mathrm{Q} 0}+\Delta_{\mathrm{I} 2}\right)\right], \\
v_{b}^{\prime}+\mathcal{H} v_{b}=-i k \phi-\frac{\tau^{\prime}}{\alpha}\left(3 i \Delta_{\mathrm{I} 1}+v_{b}\right)-\frac{i}{a} W_{B}(k) .
\end{gathered}
$$

For $\ell \geq 3$ the hierarchy of the brightness can be determined in general terms by using the recurrence relation for the Legendre polynomials reported in Eq. (A11) of the appendix:

$$
\begin{aligned}
\Delta_{\mathrm{I} \ell}^{\prime}+\tau^{\prime} \Delta_{\mathrm{I} \ell} & =\frac{k}{2 \ell+1}\left[\ell \Delta_{\mathrm{I}(\ell-1)}-(\ell+1) \Delta_{\mathrm{I}(\ell+1)}\right], \\
\Delta_{\mathrm{Q} \ell}^{\prime}+\tau^{\prime} \Delta_{\mathrm{Q} \ell} & =\frac{k}{2 \ell+1}\left[\ell \Delta_{\mathrm{Q}(\ell-1)}-(\ell+1) \Delta_{\mathrm{Q}(\ell+1)}\right] .
\end{aligned}
$$

We are now ready to compute the evolution of the various terms to a given order in the tight-coupling expansion parameter $\epsilon=\left|1 / \tau^{\prime}\right|$. After expanding the various moments of the brightness function and the velocity field in $\epsilon$

$\Delta_{\mathrm{I} \ell}=\bar{\Delta}_{\mathrm{I} \ell}+\epsilon \delta_{\mathrm{I} \ell}, \quad \Delta_{\mathrm{Q} \ell}=\bar{\Delta}_{\mathrm{Q} \ell}+\epsilon \delta_{\mathrm{Q} \ell}, \quad v_{\mathrm{b}}=\bar{v}_{\mathrm{b}}+\epsilon \delta v_{\mathrm{b}}$,

the obtained expressions can be inserted into Eqs. (5.12), (5.13), (5.14), (5.15), (5.16), and (5.17) and the evolution of the various moments of the brightness function can be found order by order.

To zeroth order in the tight-coupling approximation, the evolution equation for the baryon velocity field, i.e., Eq. (5.14), leads to

$$
\bar{v}_{b}=-3 i \bar{\Delta}_{\mathrm{I} 1},
$$

while Eqs. (5.13) and (5.16) lead, respectively, to

$$
\bar{\Delta}_{\mathrm{Q} 0}=\bar{\Delta}_{\mathrm{I} 2}+\bar{\Delta}_{\mathrm{Q} 2}, \quad \bar{\Delta}_{\mathrm{Q} 1}=0 .
$$

Finally Eqs. (5.18) and (5.19) imply

$$
9 \bar{\Delta}_{\mathrm{I} 2}=\bar{\Delta}_{\mathrm{Q} 0}+\bar{\Delta}_{\mathrm{Q} 2}, \quad 9 \bar{\Delta}_{\mathrm{Q} 2}=\bar{\Delta}_{\mathrm{Q} 0}+\bar{\Delta}_{\mathrm{I} 2} .
$$

Taking together the four conditions expressed by Eqs. (5.24) and (5.25) we have, to zeroth order in the tight-coupling approximation:

$$
\bar{\Delta}_{\mathrm{Q} \ell}=0, \quad \ell \geq 0, \quad \bar{\Delta}_{\mathrm{I} \ell}=0, \quad \ell \geq 2 .
$$

Hence, to zeroth order in the tight coupling, the relevant equations are

$$
\begin{gathered}
\bar{v}_{b}=-3 i \bar{\Delta}_{\mathrm{I} 1}, \\
\bar{\Delta}_{\mathrm{I} 0}^{\prime}+k \bar{\Delta}_{\mathrm{I} 1}=\psi^{\prime} .
\end{gathered}
$$

This means, as anticipated, that to zeroth order in the tight-coupling expansion the $\mathrm{CMB}$ is not polarized since the quadrupole moment of the brightness is vanishing. Summing up Eqs. (5.15) and (5.17) and using Eq. (5.27) in order to eliminate $\bar{v}_{\mathrm{b}}$ from the obtained expression, we get to the following equation:

$$
\begin{gathered}
(\alpha+1) \bar{\Delta}_{\mathrm{I} 1}^{\prime}+\mathcal{H} \alpha \bar{\Delta}_{\mathrm{I} 1}-\frac{k}{3} \bar{\Delta}_{\mathrm{I} 0} \\
=\frac{k}{3}(\alpha+1) \phi+\frac{i \alpha}{3} W_{B}(k) .
\end{gathered}
$$

Finally, the dipole term can be eliminated from Eq. (5.29) using Eq. (5.28). By doing so, Eq. (5.29) leads to the wanted decoupled equation for the monopole:

$$
\begin{aligned}
& \bar{\Delta}_{\mathrm{I} 0}^{\prime \prime}+\frac{\alpha^{\prime}}{\alpha+1} \bar{\Delta}_{\mathrm{I} 0}^{\prime}+\frac{k^{2}}{3(\alpha+1)} \bar{\Delta}_{\mathrm{I} 0} \\
& \quad=\left[\psi^{\prime \prime}+\frac{\alpha^{\prime}}{\alpha+1} \psi^{\prime}-\frac{k^{2}}{3} \phi\right]-\frac{\alpha}{\alpha+1} \frac{F_{\mathrm{B}}}{12 \pi \rho_{\mathrm{b}} a^{4}} .
\end{aligned}
$$

In the limit $F_{\mathrm{B}} \rightarrow 0$ the usual decoupled equation for the evolution of the monopole is recovered.

The presence of the magnetic field modifies the evolution of the monopole. Hence, also the dipole will be modified since the relation (5.28) stipulates that the monopole is a source of the dipole.

To first order in the tight-coupling limit, the relevant equations can be obtained by keeping all terms of order $\epsilon$ and by using the first-order relations to simplify the expressions. From Eq. (5.16) the condition $\delta_{\mathrm{Q} 1}=0$ can be derived; from Eqs. (5.13), (5.18), and (5.19), the following remaining conditions are obtained, respectively:

$$
\begin{gathered}
-\delta_{\mathrm{Q} 0}+\delta_{\mathrm{I} 2}+\delta_{\mathrm{Q} 2}=0, \\
\frac{9}{10} \delta_{\mathrm{I} 2}-\frac{1}{10}\left[\delta_{\mathrm{Q} 0}+\delta_{\mathrm{Q} 2}\right]=\frac{2}{5} k \bar{\Delta}_{\mathrm{I} 1}, \\
\frac{9}{10} \delta_{\mathrm{Q} 2}-\frac{1}{10}\left[\delta_{\mathrm{Q} 0}+\delta_{\mathrm{I} 2}\right]=0 .
\end{gathered}
$$

Finally from Eqs. (5.31), (5.32), and (5.33) the remaining relations are

$$
\begin{aligned}
& \delta_{\mathrm{Q} 0}=\frac{5}{4} \delta_{\mathrm{I} 2}, \\
& \delta_{\mathrm{Q} 2}=\frac{1}{4} \delta_{\mathrm{I} 2}, \\
& \delta_{\mathrm{I} 2}=\frac{8}{15} \bar{\Delta}_{\mathrm{I} 1} .
\end{aligned}
$$

Condition (5.36) can be also written

$$
\Delta_{\mathrm{I} 2}=\epsilon \delta_{\mathrm{I} 2}=\frac{8}{15} \frac{k}{\tau^{\prime}} \bar{\Delta}_{\mathrm{I} 1} .
$$


Now, from Eqs. (5.34) and (5.35), the quadrupole moment of $\Delta_{\mathrm{Q}}$ is proportional to the quadrupole of $\Delta_{\mathrm{I}}$, which is, in turn, proportional to the dipole evaluated to first order in $\epsilon$. But $\Delta_{\mathrm{Q}}$ measures exactly the degree of linear polarization of the radiation field. So, to first order in the tightcoupling expansion, the CMB is linearly polarized. Furthermore, as discussed in Eq. (5.30) the presence of the magnetic field modifies the evolution of both the monopole and the dipole. Thus, according to Eq. (5.37), also the polarization will be modified.

All the solutions discussed in the previous sections can be used to determine the initial conditions for the evolution of the brightness using the tight-coupling expansion. Consider, as a possible example, the case when initial conditions are set deep within the radiation epoch. In this case, recalling Eq. (5.8), Eq. (5.30) can be written, for $\alpha \ll 1$, as

$$
\Delta_{0}^{\prime \prime}+\omega^{2} \Delta_{0}=-\omega^{2}(\phi+\psi)-\frac{F_{\mathrm{B}}}{16 \pi \bar{\rho}_{\gamma}},
$$

where $\Delta_{0}=\bar{\Delta}_{\mathrm{I} 0}-\psi$ and $\omega=k / \sqrt{3}$; we also posit $a^{4} \rho_{\gamma}=\bar{\rho}_{\gamma}$. The solution of Eq. (5.38) can be easily obtained in terms of arbitrary integration constants. These constants are fixed by specifying the initial conditions for the velocity field $v_{\mathrm{b}}$, which determines, according to Eq. (5.23), the value of the dipole. Suppose, for instance, to be interested in the case of quasiadiabatic initial conditions in the presence of the reduced Lorentz force. Then, from Eqs. (3.59) and (5.23), recalling that $\theta_{\mathrm{b}}=i k v_{b}$,

$$
\bar{\Delta}_{\mathrm{I} 1}=\frac{k \phi_{0}}{6} \eta+\frac{F_{\mathrm{B}}}{12 \pi \rho_{\mathrm{b}} a^{3}} .
$$

From Eq. (5.17) the initial condition for $\Delta_{0}$ can be determined to be

$$
\Delta_{0} \simeq-\frac{3}{2}\left(1+\frac{4}{15} R_{\nu}\right) \phi_{0}+\frac{R_{\nu}}{5} \Omega_{\mathrm{B}}-\frac{3}{5} \sigma_{\mathrm{B}} .
$$

The same strategy can be applied to more specific cases, such as the one where the scale factor interpolates between a radiation-dominated phase and a matterdominated phase, as discussed in Eq. (4.29). In this case the solution of Eq. (5.30) will be more complicated but always analytically tractable.

\section{CONCLUDING REMARKS}

In this paper a systematic treatment of scalar perturbations has been discussed in the presence of a fully inhomogeneous magnetic field. No specific configuration has been assumed and the results are, in this sense, rather general. Large-scale magnetic fields have been described in a fully consistent one-fluid MHD approach in curved space-time, which is particularly suitable for the analysis of the low-frequency part of the plasma spectrum. In this approach the charged components of the plasma (baryons and electrons) are in thermal equilibrium at a common temperature. The neutral components of the plasma (photons, neutrinos and CDM particles) are not affected directly by the presence of the magnetic fields. However, since magnetic fields gravitate and appear in the perturbed Einstein equations, also the initial conditions for the evolution of the neutral species are modified in a specific fashion. Since in MHD the Ohmic current is solenoidal, the baryon evolution equation is affected by a reduced Lorentz force term whose characteristic form is well known in flat-space MHD. The combination of these different effects leads to a set of initial conditions for the $\mathrm{CMB}$ anisotropies, which is rather different from the standard adiabatic (or isocurvature) modes.

The main new results of the present analysis can be summarized as follows:

(i) the problem of initial conditions for magnetized CMB anisotropies has been solved both in the conformally Newtonian gauge (more useful for theoretical calculations) and in the synchronous gauge (more appropriate for numerical discussions);

(ii) if the curvature fluctuations are adiabatic, magnetic fields modify the conventional adiabatic mode in a computable way and, as a consequence, the whole system of initial conditions for CMB anisotropies is modified;

(iii) for the modified adiabatic mode, deep outside the horizon, the density contrasts still satisfy the adiabaticity condition; however, as the horizon is crossed, a small nonadiabatic component develops;

(iv) if the fluctuations are isocurvature from the beginning, magnetic fields modify quantitatively their amplitude, as shown in the case of the baryon isocurvature mode;

(v) the tight-coupling expansion has been revisited in the presence of a fully inhomogeneous magnetic field;

(vi) it has been shown that, because of the reduced Lorentz force, both the zeroth and first order in the tight-coupling expansion are modified and this allows the initial conditions for the evolution of the brightness functions to be computed reliably in both the adiabatic and isocurvature cases when fully inhomogeneous magnetic fields are present.

It is appropriate to conclude with some remarks concerning the interplay between large-scale magnetic fields and CMB physics. In various investigations, limits on the magnetic field intensity are obtained on the basis of CMB considerations. Most of the time these limits refer to specific configurations. These discussions are certainly valuable; however, the limits obtained should always be associated with a specific set of analytical initial conditions. As far as scalar fluctuations are concerned, this 
was not done up to now. An example is Ref. [8] where no specific analysis of the initial condition problem was presented, but rather stringent limits were claimed.

We must bear in mind that the analysis of CMB anisotropies requires the determination of a sizeable set of parameters. If the magnetic field were the only unknown, the task would be easier. However, this is unfortunately not the case. Hence, even within a specific magnetic field configuration, the limits should always refer to the specific set of initial conditions assumed in the analysis, as is normally done in the case when magnetic fields are absent. We do hope that future analyses along these directions may take the results of the present paper as a useful format for the systematic investigation of the effects of large-scale magnetic fields on the CMB anisotropies.

\section{APPENDIX: BOLTZMANN HIERARCHY}

As discussed in the bulk of the paper neutrinos are collisionless particles. In the following we are going to discuss the collisionless Boltzmann equation following the notation discussed in the present paper. The phasespace distribution of massless particles (massless neutrinos or photons) is defined to be

$$
f\left(x^{i}, P_{i}, \eta\right) d x^{1} d x^{2} d x^{3} d P_{1} d P_{2} d P_{3}=d \mathcal{N},
$$

giving the number of particles in a differential volume of phase space. The function appearing in Eq. (A1) is scalar under canonical transformations, $P_{i}$ is the conjugate momentum. To derive and use the Boltzmann equation in curved space-time it is more convenient to work directly with the modulus $q$ and the direction $n_{i}$ of the comovingthree-momentum $q_{i}$ (where $q_{i}=q n_{i}$ with $n_{i} n^{i}=1$ ). Denoting the longitudinal degrees of freedom of the perturbed geometry as in Eq. (2.6) and adopting the metric signature of Eq. (2.2), the relation between the comoving-three-momentum and the components of the conjugate momentum are $P_{i}=-q_{i}(1-\psi)$ and $P_{0}=q(1+\phi)$.

The total variation of the distribution function can be written as

$$
\frac{D f}{D \eta}=\frac{\partial f}{\partial \eta}+\frac{\partial x^{i}}{\partial \eta} \frac{\partial f}{\partial x^{i}}+\frac{\partial f}{\partial q} \frac{\partial q}{\partial \eta}+\frac{\partial f}{\partial n_{i}} \frac{\partial n^{i}}{\partial \eta}=\left(\frac{\partial f}{\partial \eta}\right)_{\text {coll }},
$$

where the collisional term has been kept for later convenience, but it is zero in the case of neutrinos. The collisionless part of Eq. (A2) can be perturbed around a configuration of thermal equilibrium by splitting the phase-space distribution as

$$
f\left(x^{i}, P_{j}, \eta\right)=f_{0}(q)\left[1+f^{(1)}\left(x^{i}, q, n_{j}, \eta\right)\right],
$$

where $f_{0}(q)$ is the unperturbed phase-space distribution, which only depends upon the comoving-threemomentum.
Inserting Eq. (A3) into Eq. (A2), only the terms that are first order will be kept. Since the unperturbed phasespace distribution only depends upon the comovingthree-momentum, Eq. (A2) becomes

$$
\frac{\partial f^{(1)}}{\partial \eta}+n^{i} \frac{\partial f^{(1)}}{\partial x^{i}}+\frac{\partial \ln f_{0}}{\partial \ln q}\left[\psi^{\prime}-n_{i} \partial^{i} \phi\right]=\frac{1}{f_{0}}\left(\frac{\partial f}{\partial \eta}\right)_{\text {coll }},
$$

where the geodesic equation has been used in order to obtain the expression of the time derivative of $q$ to first order in the amplitude of the metric perturbations, i.e.,

$$
\frac{d q}{d \eta}=q \psi^{\prime}-q n_{i} \partial^{i} \phi
$$

Up to this point the derivation is valid for both photons and massless neutrinos.

\section{Massless neutrinos and photons}

Going now to Fourier space and defining the reduced phase-space distribution for massless neutrinos as

$$
\mathcal{F}_{\nu}(\vec{k}, \hat{n}, \eta)=\frac{\int q^{3} d q f_{0} f^{(1)}}{\int q^{3} d q f_{0}}
$$

Eq. (A4) becomes, in the absence of collision term,

$$
\frac{\partial \mathcal{F}_{\nu}}{\partial \eta}+i k \mu \mathcal{F}_{\nu}=4\left(\psi^{\prime}-i k \mu \phi\right)
$$

where $\mu=\hat{n} \cdot \hat{k}$. The factor 4 appearing in Eq. (A7) follows from the explicit expression of the Fermi-Dirac phase-space distribution and observing

$$
\int q^{3} d q \frac{\partial \ln f_{0}}{\partial \ln q}=-4 \int q^{3} d q f_{0} .
$$

The reduced phase-space distribution can be expanded in series of Legendre polynomials as

$$
\mathcal{F}_{\nu}(\vec{k}, \hat{n}, \eta)=\sum_{\ell}(-i)^{\ell}(2 \ell+1) \mathcal{F}_{\nu \ell}(\vec{k}, \eta) P_{\ell}(\mu) .
$$

Equation (A9) will now be inserted into Eq. (A7). The orthonormality relation for Legendre polynomials,

$$
\int_{-1}^{1} P_{\ell}(\mu) P_{\ell^{\prime}}(\mu)=\frac{2}{2 \ell+1} \delta_{\ell \ell^{\prime}}
$$

together with the well-known recurrence relation

$$
(\ell+1) P_{\ell+1}(\mu)=(2 \ell+1) \mu P_{\ell}(\mu)-\ell P_{\ell-1}(\mu)
$$

allows one to get a hierarchy of equations to be obtained for the various multipole moments. The procedure is to take the various moments of both sides of Eq. (A7). In doing so, expressions like 


$$
\begin{aligned}
i k \int_{-1}^{1} \mu P_{\ell^{\prime}}(\mu) \mathcal{F}_{\nu} d \mu= & 2 i k\left[(-i)^{\ell^{\prime}+1} \frac{\ell^{\prime}+1}{2 \ell^{\prime}+1} F_{\nu\left(\ell^{\prime}+1\right)}\right. \\
& \left.+(-i)^{\ell^{\prime}-1} \frac{\ell^{\prime}}{2 \ell^{\prime}+1} F_{\nu\left(\ell^{\prime}-1\right)}\right]
\end{aligned}
$$

will appear; they can be evaluated by using Eqs. (A10) and (A11). The full form of the Boltzmann hierarchy is then

$$
\begin{gathered}
\mathcal{F}_{\nu 0}^{\prime}=-k \mathcal{F}_{\nu 1}+4 \psi^{\prime}, \\
\mathcal{F}_{\nu 1}^{\prime}=\frac{k}{5}\left[\mathcal{F}_{\nu 0}-2 \mathcal{F}_{\nu 2}\right]+\frac{4}{3} k \phi, \\
\mathcal{F}_{\nu \ell}^{\prime}=\frac{k}{2 \ell+1}\left[\ell \mathcal{F}_{\nu,(\ell-1)}-(\ell+1) \mathcal{F}_{\nu(\ell+1)}\right] .
\end{gathered}
$$

Equation (A14) holds for $\ell \geq 2$.

The components of the energy-momentum tensor can be connected with the monopole and dipole of the distribution function

$$
T_{\mu}^{\nu}=-\int \frac{d^{3} P}{\sqrt{-g}} \frac{P_{\mu} P^{\nu}}{P^{0}} f\left(x^{i}, P_{j}, \eta\right) .
$$

Recalling the connection between conjugate momenta and comoving-three-momenta we get, to zeroth order:

$$
\rho_{\nu}=\frac{1}{a^{4}} \int d^{3} q q f_{0}(q),
$$

while to first order the density contrast (monopole), the peculiar velocity field (dipole) and the quadrupole become

$$
\begin{gathered}
\delta_{\nu}=\frac{1}{4 \pi} \int d \Omega \mathcal{F}_{\nu}(\vec{k}, \hat{n}, \eta), \\
\theta_{\nu}=\frac{3 i}{16 \pi} \int d \Omega(\vec{k} \cdot \hat{n}) \mathcal{F}_{\nu}(\vec{k}, \hat{n}, \eta)=\frac{3}{4} k \mathcal{F}_{\nu 1}, \\
\sigma_{\nu}=-\frac{3}{16 \pi} \int d \Omega\left[(\vec{k} \cdot \hat{n})^{2}-\frac{1}{3}\right] \mathcal{F}_{\nu}(\vec{k}, \hat{n}, \eta)=\frac{\mathcal{F}_{\nu 2}}{2} .
\end{gathered}
$$

Inserting Eqs. (A17) and (A19) into Eqs. (A13) and (A14), the system following from the perturbation of the covariant conservation equations can be partially recovered

$$
\begin{gathered}
\delta_{\nu}^{\prime}=-\frac{4}{3} \theta_{\nu}+4 \psi^{\prime}, \\
\theta_{\nu}^{\prime}=\frac{k^{2}}{4} \delta_{\nu}-k^{2} \sigma_{\nu}+k^{2} \phi, \\
\sigma_{\nu}^{\prime}=\frac{4}{15} \theta_{\nu}-\frac{3}{10} k \mathcal{F}_{\nu 3},
\end{gathered}
$$

with the important addition of the quadrupole [appearing in Eq. (A20)] and of the whole Eq. (A22), which couples the quadrupole, the peculiar velocity field, and the octupole $\mathcal{F}_{\nu 3}$. Equation (A22) is important. After neutrino decoupling, when initial conditions are set, $\mathcal{F}_{\nu 3}=0$.

Equation (A4) can also be made explicit in the case of photons. In order to do so the collision term should be specified. Before writing down the Boltzmann equation for the photons it is useful, in order to match with the standard notations, to define the photon brightness perturbation, conventionally denoted by $\Delta$ :

$$
f\left(x^{i}, q, n_{j}, \eta\right)=f_{0}\left(\frac{q}{1+\Delta}\right),
$$

where $f_{0}(q)$, i.e., the unperturbed phase-space distribution, denotes now the Bose-Einstein distribution. Comparing Eq. (A3) with the expression given in Eq. (A23) and expanding for $\Delta<1$, the two definitions are connected as

$\Delta=-f^{(1)}\left(\frac{\partial \ln f_{0}}{\partial \ln q}\right)^{-1}, \quad \mathcal{F}_{\gamma}=-\Delta \frac{\int q^{3} d q \frac{\partial \ln f_{0}}{\partial \ln q}}{\int q^{3} d q f_{0}}=4 \Delta$,

where $\mathcal{F}_{\gamma}$ is the reduced phase-space distribution for the photons defined in full analogy with the case of the massless neutrinos, i.e., Eq. (A6). The second identity of Eq. (A24) follows from the first and from Eq. (A8). Using the relation between the brightness perturbation and the phase-space distribution, the collisionless part of the Boltzmann equation can be written as

$$
\Delta^{\prime}+i k \mu(\Delta+\phi)=\psi^{\prime} .
$$

The brightness perturbation, as defined in Eq. (A23), corresponds physically to a perturbation in the first Stokes parameter (conventionally denoted by I), i.e., to the sum of the squared amplitudes of the radiation field. By specifying the collision term, the full form of the Boltzmann equation for photons can be written as

$$
\begin{gathered}
\Delta_{\mathrm{I}}^{\prime}+i k \mu \Delta_{\mathrm{I}}=\left(\psi^{\prime}-i k \mu \phi\right)+\tau^{\prime}\left[-\Delta_{\mathrm{I}}+\Delta_{\mathrm{I} 0}\right. \\
\left.+\mu v_{\mathrm{b}}-\frac{1}{2} P_{2}(\mu) S_{0}\right], \\
\Delta_{\mathrm{Q}}^{\prime}+i k \mu \Delta_{\mathrm{Q}}=\tau^{\prime}\left\{-\Delta_{\mathrm{Q}}+\frac{1}{2}\left[1-P_{2}(\mu)\right] S_{0}\right\}, \\
\Delta_{\mathrm{U}}^{\prime}+i k \mu \Delta_{\mathrm{U}}=-\tau^{\prime} \Delta_{\mathrm{U}} .
\end{gathered}
$$

Concerning Eqs. (A26)-(A28) a few comments are in order. The brightness functions $\Delta_{\mathrm{Q}}$ and $\Delta_{\mathrm{U}}$ correspond, respectively, to the Stokes parameters $Q$ and U. The parameter $\mathrm{Q}$, being the difference of the squares of the amplitudes of the radiation field, is sensitive to the linearly polarized radiation. Since Q and U (unlike I and V) change under rotations, once $\mathrm{Q}$ is included also $\mathrm{U}$ must follow. The source term appearing in Eq. (A26), $S_{0}$ is, within our conventions, 


$$
S_{0}=\Delta_{\mathrm{I} 2}+\Delta_{\mathrm{Q} 0}+\Delta_{\mathrm{Q} 2},
$$

where, with obvious notations, $\Delta_{\mathrm{I} \ell}$ denotes the $\ell$ th multipole of $\Delta_{\mathrm{I}}$ and similarly for $\Delta_{\mathrm{Q}}$. The peculiar velocity field for the baryons has been written as

$$
v_{\mathrm{b}}=\frac{\theta_{\mathrm{b}}}{i k} .
$$

The notation $v_{\mathrm{b}}$ is preferred here in view of the application to the tight-coupling expansion of the Boltzmann hierarchy for the photon brightness. Finally, $\tau^{\prime}=$ $x_{e} n_{e} \sigma_{\mathrm{T}}\left(a / a_{0}\right)$ is, as previously introduced, the differential optical depth for Thompson scattering. The evolution equation for $v_{\mathrm{b}}$ can be easily obtained, for instance by Fourier transforming Eq. (4.26) and by using Eq. (A30):

$$
v_{\mathrm{b}}^{\prime}+\mathcal{H} v_{\mathrm{b}}+i k \phi+\frac{\tau^{\prime}}{\alpha}\left(3 i \Delta_{\mathrm{I} 1}+v_{b}\right)=-\frac{i}{4 \pi k} \frac{F_{\mathrm{B}}(k)}{a^{4} \rho_{\mathrm{b}}},
$$

having defined

$$
\alpha=\frac{3}{4} \frac{\rho_{\mathrm{b}}}{\rho_{\mathrm{r}}} .
$$

Equations (A26)-(A28) and (A31) are discussed in Sec. IV.
[1] Ya. Zeldovich and I. Novikov, The Structure and Evolution of the Universe (Chicago University, Chicago, 1971), Vol. 2.

[2] Ya. Zeldovich, Sov. Phys. JETP 21, 656 (1965).

[3] J. M. Bardeen, Phys. Rev. D 22, 1882 (1980).

[4] W. Hu and N. Sugiyama, Astrophys. J. 471, 542 (1996); 444, 489 (1995).

[5] C.-P. Ma and E. Bertschinger, Astrophys. J. 455, 7 (1995).

[6] W. Press and E. Vishniac, Astrophys. J. 239, 1 (1980); 236, 323 (1980).

[7] T. Kahniashvili, astro-ph/0405184.

[8] S. Koh and C. H. Lee, Phys. Rev. D 62, 083509 (2000).

[9] A. Mack, T. Kahniashvili, and A. Kosowsky, Phys. Rev. D 65, 123004 (2002).

[10] A. Lewis, Phys. Rev. D 70, 043011 (2004).

[11] J. Barrow, P. Ferreira, and J. Silk, Phys. Rev. Lett. 78, 3610 (1997).

[12] J. Adams, U. Danielsson, D. Grasso, and H. Rubinstein, Phys. Lett. B 388, 253 (1996).

[13] K. Subramanian and J. Barrow, Mon. Not. R. Astron. Soc. 335, L57 (2002).

[14] K. Subramanian, T. Seshadri, and J. Barrow, Mon. Not. R. Astron. Soc. 344, L31 (2003).

[15] G. Chen, P. Mukherjee, T. Kahniashvili, B. Ratra, and Y. Wang, Astrophys. J. 611, 655 (2004).

[16] A. Kosowsky and A. Loeb, Astrophys. J. 461, 1 (1996).

[17] D. Harari, J. Hayward, and M. Zaldarriaga, Phys. Rev. D 55, 1841 (1997).

[18] M. Giovannini, Phys. Rev. D 56, 3198 (1997).

[19] C. Scoccola, D. Harari, and S. Mollerach, Phys. Rev. D 70, 063003 (2004).

[20] M. Giovannini, Int. J. Mod. Phys. D 13, 391 (2004).

[21] C.G. Tsagas and J.D. Barrow, Classical Quantum Gravity 14, 2539 (1997); 15, 3523 (1998).

[22] C. Tsagas and R. Maartens, Phys. Rev. D 61, 083519 (2000); Classical Quantum Gravity 17, 2215 (2000).
[23] N. A. Krall and A.W. Trivelpiece, Principles of Plasma Physics (San Francisco Press, San Francisco, 1986).

[24] T. J. M. Boyd and J. J. Sanderson, The Physics of Plasmas (Cambridge University, Cambridge, England, 2002).

[25] D. Biskamp, Non-linear Magnetohydrodynamics (Cambridge University, Cambridge, England, 1994).

[26] A. Brandenburg, K. Enqvist, and P. Olesen, Phys. Rev. D 54, 1291 (1996).

[27] A. Brandenburg, K. Enqvist, and P. Olesen, Phys. Lett. B 392, 395 (1997).

[28] P. Olesen, Phys. Lett. B 398, 321 (1997).

[29] P. Olesen, in Primordial Magnetic Fields and Their Development (Applied Field Theory), NATO ASI, Ser. B, Vol. 366 (Plenum, New York, 1998), p. 159.

[30] K. Enqvist, Int. J. Mod. Phys. D 7, 331 (1998).

[31] K. Holcomb and T. Tajima, Phys. Rev. D 40, 3809 (1989); C. P. Dettmann, N. E. Frankel, and V. Kowalenko, Phys. Rev. D 48, 5655 (1993).

[32] R. M. Gailis, C. P. Dettmann, N.E. Frankel, and V. Kowalenko, Phys. Rev. D 50, 3847 (1994).

[33] R. M. Gailis, N.E. Frankel, and C.P. Dettmann, Phys. Rev. D 52, 6901 (1995).

[34] I. Wasserman, Astrophys. J. 224, 337 (1978).

[35] P. Coles, Comments Astrophys. 16, 45 (1992).

[36] M. Bucher, K. Moodley, and N. Turok, Phys. Rev. Lett. 87, 191301 (2001).

[37] L. Campanelli, Phys. Rev. D 70, 083009 (2004).

[38] M. Giovannini, Phys. Rev. D 61, 063004 (2000); 61, 063502 (2000).

[39] M. Giovannini and M. Shaposhnikov, Phys. Rev. Lett. 80, 22 (1998); Phys. Rev. D 57, 2186 (1998).

[40] V.F. Mukhanov, H. A. Feldman, and R. H. Brandenberger, Phys. Rep. 215, 203 (1992).

[41] P. J.E. Peebles and J.T. Yu, Astrophys. J. 162, 815 (1970). 